\title{
A Popov-theory-based survey on digital control of infinite-dimensional systems with unboundedness
}

\author{
Florin Dan Barb and Willem L. De Koning \\ Delft University of Technology, Department of Technical Mathematics and \\ Informatics, Mekelweg 4. 2628 CD Delft. The Netherlands \\ VLAD IONESCU \\ Polytechnic Institute of Bucharest, Department of Automatic Control, \\ 313 Splaiul Independentei. 77206 Bucharest, Romania
}

[Received 14 June 1994 and in revised form 11 December 1994]

\begin{abstract}
This paper represents a Popov-theory-based assessment of the current status of the digital control of infinite-dimensional systems with certain unboundedness in control and/or observation, summarizing some results and indicating which, in the authors' opinion, would be the promising directions for future research.
\end{abstract}

\section{Introduction}

Control by computers has become an everyday reality for more than a decade. With the advent and proliferation of microcomputers, the role played by digital control design techniques has become increasingly more important. In many industrial settings, the designer has to control physical entities such as temperature and fluid flow, and appropriate modelling often leads to distributed-parameter systemssystems which are defined on Hilbert spaces. It has become necessary, therefore, to develop extensions of many of the 'classical' digital control strategies for finitedimensional systems to an infinite-dimensional setting. A generalization, to infinitedimensional systems with bounded operators, of the finite-dimensional results on the digital LQ optimal-control problem was achieved more than 20 years ago by Lee et al. [25]. Since then, various finite-dimensional control problems have been addressed and solved; as one would expect, various approaches to and theories about those control problems have been taken and constructed. We refer here to the $\mathrm{H}^{2}$ optimal control problem when considered in the deterministic case, the LQG optimal-control problem when considered in the stochastic sense, and the $\mathrm{H}^{x}$ suboptimal-control problems. In this paper, we consider the aforementioned problems in the infinite-dimensional setting, and our approach to control is a digital one. From the rather wide variety of control theories that have emerged up to this time, we shall focus our attention upon the so-called Popov theory. Let us begin by explaining why we have chosen this option, i.e. why a discrete Popov-theory approach to the digital control of DP systems is a valuable one. There are two main reasons. 
(i) Among different results emerging from Popov's positiveness theory [27]-a theory with origins in the work of Kalman [24] and Yakubovitch [38]-the one that establishes the connections between the properties of a quadratic cost functional and the existence of a stabilizing solution to a certain Riccati equation is probably most relevant. This is especially true since there are, probably, few control-theory concepts that have been more extensively studied than Riccati equations. For more than three decades, their application to stability, LQ optimal control, and more recently $\mathrm{H}^{2}$ optimal control and $\mathrm{H}^{x}$ optimal control, has formed a strong basis for the development of a huge control-theory literature.

(ii) The second motivation for our choice of the discrete Popov-theory approach is the possibility of replacing the positivity condition of the Popov function with a more general one, given by the invertibility of a certain Toeplitz-like operator. The reader is referred to the original work of Ionescu \& Weiss [21] and its extensions [18]. This new direction has proved to be rich enough to incorporate game-theoretic situations (see [9]) and to permit one to write down the solution to the $\mathrm{H}^{\propto}$ control problem.

Classical optimal control theory of distributed-parameter systems assumes boundedness of control and observation operators. However, most of the interesting infinitedimensional control systems encountered in practice arise in a different way. The next example fully supports this statement.

EXAMPLE 1.1 The temperature distribution of a unit-length heated rod with point heat control and point temperature measurement is described by the following PDE model:

$$
\begin{array}{r}
\frac{\partial z(x, t)}{\partial t}=\frac{\partial^{2} z(x, t)}{\partial x^{2}}+\delta_{x_{0}}(x) u(t), \quad z(x, 0)=z_{0}(x), \\
{\left[\frac{\partial z(x, t)}{\partial t}\right]_{x=0}=\left[\frac{\partial z(x, t)}{\partial t}\right]_{x=1}=0, \quad y(t)=z\left(x_{1}, t\right),}
\end{array}
$$

where $z(x, t)$ is the temperature distribution, $x \in[0,1]$ the distance along the rod, $u(t)$ the control, and $y(t)$ the observation. In this example, the observation operator is the temperature measurement at a point $x_{1}$, formally defined by

$$
C z=z\left(x_{1}, \cdot\right) \text {, }
$$

which is unbounded on the state space $\mathcal{X}=\mathrm{L}_{2}[0,1]$; it is not defined on all of $\mathrm{L}_{2}[0,1]$. Since we have modelled the control operator in terms of the delta distribution at $x_{0}$, it is clear that the control mapping is not bounded either, and the control system from this example has unbounded control and observation operators.

Let us now explain why the digital control of systems with unboundedness is a difficult and interesting problem. There are three common ways to design a digital control system.

(i) The first way is to prepare a continuous-time design followed by a digital implementation, i.e. the time-discretized controller is connected via $A-D$ and $D-A$ devices to the original continuous-time plant. Besides the general disadvantage of 
the sampling frequency not being a designable parameter in such a design process, a particular disadvantage arises in the case of infinite-dimensional systems with unboundedness, which makes this method unapplicable. This statement will be clarified in the next paragraph. Van Keulen [34] gave a complete generalization for the Pritchard-Salamon class of infinite-dimensional systems [28] of the early Doyle et al. results on $\mathrm{H}^{\infty}$ optimal control [16]. Besides the full generalization of the above-mentioned results, he showed that the system acting as a controller that minimizes the $\mathbf{H}^{x}$ norm of the closed-loop system belongs also to the PritchardSalamon class. Hence the digital implementation of such a controller requires discretizing a Pritchard-Salamon system-a task which (as shown in Barb et al. [5]) is not always possible unless certain restrictions on the initial state are imposed to guarantee the smoothness of the system output function.

(ii) The second way is to discretize the plant and to formulate a discrete-time design. This method is also not the best one could hope for: besides the obvious disadvantage of ignoring intersampling behaviour, it has the same drawback as the first-mentioned method-that of having to discretize a Pritchard-Salamon system.

(iii) The third way is through direct digital design, treating the control system as a sampled-data system. Since sampled-data systems are time-varying systems (actually continuous-time $T$-periodic systems), it is rather obvious that the design process will be more complicated than in the time-invariant case.

With respect to the direct digital design approach, let us note that there are several sources of difficulty that one has to overcome in the design process, such as

- the structural constraint on the controller $\mathrm{H}_{T} \Sigma_{\mathrm{K}} \mathrm{S}_{T}$, where $\mathrm{H}_{T}$ and $\mathrm{S}_{T}$ are the zeroth-order hold and sampler, and $\Sigma_{\mathrm{K}}$ is the discrete-time controller;

- the fact that the controller $\mathrm{H}_{T} \Sigma_{\mathrm{K}} \mathrm{S}_{T}$ is not time-invariant, despite $\Sigma_{\mathrm{K}}$ being so;

- the hybrid nature of the overall control system: the state of the plant evolves in continuous time, while the controller state evolves in discrete-time, and hence they are not defined over the same time set.

One approach to direct digital design is given by the general framework for periodic continuous-time systems as proposed by Bamieh \& Pearson [7]. The main tool used in this framework is the so-called lifting technique originated in the work of Yamamoto [39]; essentially, lifting means to represent a linear time-varying (periodic) continuoustime system by a linear time-invariant discrete-time one such that the periodicity of the first system is reflected in the shift invariance of the second. + Working in such a framework as proposed in [7], one can reduce the digital control problem to an equivalent discrete-time control problem for its lifted counterpart, in the sense that a solution to the latter is also a solution to the former. Sections 4 and 5 in [7] give comprehensive treatment of this subject. In the present paper, we adopt the same framework. Unlike Tadmor who gives the solution to the problem of $\mathrm{H}^{x}$ sampleddata control in continuous time in terms of three Riccati equations (see [33]), we deal with a discrete-time control problem on abstract Hilbert spaces, and hence we

+This idea was 'floating' in the community of researchers on digital control. Several other authors have developed a similar lifting theory in the same period of time. The reader is referred also to Francis \& Tannenbaum [8]. 


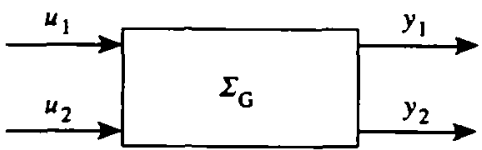

Fig. 1. The generalized plant.

shall give the solution in terms of discrete-time Riccati equations. The price we pay, specific to lifting, is that we have to work with signals taking values in function spaces (if the original signal is an $\mathbb{R}$-valued function, then its lifted counterpart takes values in $\mathrm{L}_{2}(0, T ; \mathbb{R})$; see [7] for more details on lifting). For such a discrete-time control problem, the discrete Popov theory seem to be ideal tool which enables us to write down the solution to the $\mathrm{H}^{\propto}$ digital control problem.

\subsection{The basic control problem}

In the first part of this introductory section, we chose the control theory on which we shall focus in this paper. We mentioned the main control problem addressed in the literature, which will be considered from here on. Let us now be more precise about this by formulating it mathematically. Suppose we have an infinite-dimensional plant $\Sigma_{\mathrm{G}}$ (two inputs and two outputs) as shown in Fig. 1 and a controller $\Sigma_{\mathrm{K}}$ as in Fig. 2.

We interconnect the controller and the plant, so that

$$
\nu(\cdot)=y_{2}(\cdot), \quad \zeta(\cdot)=u_{2}(\cdot),
$$

and we obtain the closed-loop structure depicted in Fig. 3.

In our set-up, $u_{1}$ is the disturbance input, $y_{1}$ the controlled output, $u_{2}$ the control input, and $y_{2}$ the measured output. The problem we want to solve is that of finding a controller $\Sigma_{\mathrm{K}}$ which 'stabilizes' the plant $\Sigma_{\mathrm{G}}$ and which makes the influence of the disturbance input on the controlled output minimal in some sense. Let us be more precise about those two concepts introduced above. Let $s$ denote either the differential operator $x \mapsto \dot{x}$ (in continuous time) or the forward unit shift operator $x \mapsto x(\cdot+1)$ in the discrete-time case. We shall assume that we have a state-space description of the plant

$$
\Sigma_{\mathrm{G}}\left\{\begin{array}{l}
(s x)(t)=A x(t)+B_{1} u_{1}(t)+B_{2} u_{2}(t), \\
y_{1}(t)=C_{1} x(t)+D_{12} u_{2}(t), \\
y_{2}(t)=C_{2} x(t)+D_{21} u_{1}(t),
\end{array}\right.
$$

and of the controller

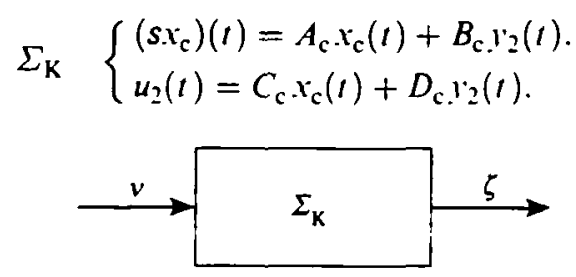

Fig. 2. The controller. 


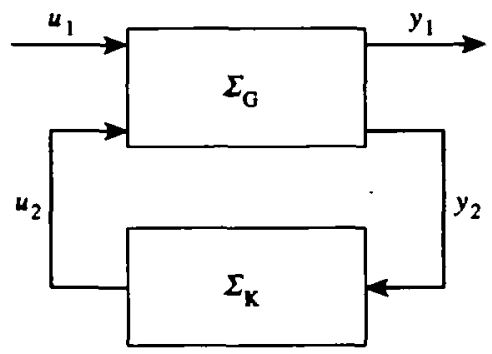

Fig. 3. The closed-loop configuration.

where $A \in \mathfrak{L}(\mathcal{X}), \quad B_{1} \in \mathfrak{I}\left(\mathcal{U}_{1}, \mathcal{X}\right), \quad B_{2} \in \mathfrak{I}\left(\mathcal{U}_{2}, \mathcal{X}\right), \quad C_{1} \in \mathfrak{I}\left(\mathcal{X}, \mathcal{Y}_{1}\right), \quad C_{2} \in \mathfrak{Q}\left(\mathcal{X}, \mathcal{Y}_{2}\right)$, $D_{12} \in \mathfrak{I}\left(\mathcal{U}_{2}, \mathcal{Y}_{1}\right), D_{21} \in \mathfrak{I}\left(\mathcal{U}_{1}, \mathcal{Y}_{2}\right), A_{\mathrm{c}} \in \mathfrak{I}\left(\mathcal{X}_{\mathrm{c}}\right), B_{\mathrm{c}} \in \mathfrak{I}\left(\mathcal{Y}_{2}, \mathcal{X}_{\mathrm{c}}\right), C_{\mathrm{c}} \in \mathfrak{I}\left(\mathcal{X}_{\mathrm{c}}, \mathcal{U}_{2}\right)$, and $D_{\mathrm{c}} \in \mathfrak{I}\left(\mathcal{Y}_{2}, \mathcal{U}_{2}\right)$ are linear time-invariant operators on the real separable Hilbert spaces $\mathcal{X}, \mathcal{U}_{1}, \mathcal{U}_{2}, \mathcal{Y}_{1}, \mathcal{Y}_{2}$, and $\mathcal{X}_{\mathrm{c}}$, respectively.

We shall say that $\Sigma_{\mathrm{K}}$ stabilizes $\Sigma_{\mathrm{G}}$ if the linear system from $u_{1}$ to $y_{1}$ is exponentially stable (in the continuous-time situation) or power stable (in the discrete-time case). This means that the closed-loop system dynamics is governed by a resultant $A$-operator

$$
A_{\mathrm{R}}=\left[\begin{array}{cc}
A+B_{2} D_{\mathrm{c}} C_{2} & B_{2} C_{\mathrm{c}} \\
B_{\mathrm{c}} C_{2} & A_{\mathrm{c}}
\end{array}\right]
$$

which is exponentially stable for the continuous case or power stable for the discrete case.

The influence of the disturbance input on the controlled output can be measured in several ways. Depending upon which one we choose, we deal with different control problems. The basic control problem we shall consider in this paper which fits in the framework depicted in Fig. 3 is presented in the following subsection.

1.1.1 The $\mathrm{H}^{x}$ control problem. Let $G$ and $K$ denote the transfer functions of $\Sigma_{\mathrm{G}}$ and $\Sigma_{\mathrm{K}}$, respectively and consider the partition on $G$ induced by its two-input and two-output structure:

$$
\left[\begin{array}{l}
v_{1} \\
y_{2}
\end{array}\right]=\left[\begin{array}{ll}
G_{11} & G_{12} \\
G_{21} & G_{22}
\end{array}\right]\left[\begin{array}{l}
u_{1} \\
u_{2}
\end{array}\right]
$$

Let the controller be given by

$$
u_{2}=K y_{2} \text {. }
$$

For such a control structure as the one depicted in Fig. 1, the transfer function from the disturbance input to the controlled output, $G_{1}$, is defined as the linear fractional transformation of the plant and controller combined:

$$
G_{1} \triangleq G_{11}+G_{12} K\left(I-G_{22} K\right)^{-1} G_{21} \text {, }
$$

where it is assumed that $I-G_{22} K$ is boundedly invertible. Since the controller achieves exponential stability of the closed-loop system, it follows that $G_{1}$ belongs to the Hardy space $\mathrm{H}^{x}\left(\mathfrak{L}\left(\mathcal{U}_{1}, \mathcal{Y}_{1}\right)\right)$, the space of $\mathfrak{L}\left(\mathcal{U}_{1}, \mathcal{Y}_{1}\right)$-valued functions which are bounded and analytic in the right half plane

$$
\mathbb{C}_{+}=\{s \in \mathbb{C}: \operatorname{Re} s>0\} \text {, }
$$


in the continuous-time case, or outside the closed unit disc

$$
\mathrm{U}_{1}(0)=\{z \in \mathbb{C}:|z| \leqslant 1\},
$$

in the discrete-time case. An appropriate measure of the influence of $u_{1}$ on $y_{1}$ is the $\mathrm{H}^{x}$ norm of $G_{1}$ defined by

$$
\left\|G_{1}\right\|_{\infty} \triangleq \begin{cases}\sup _{\operatorname{Re} s>0}\left\|G_{1}(s)\right\|_{\mathfrak{R}\left(u_{1}, y_{1}\right)} & \text { in the continuous-time case, } \\ \sup _{\|=\|>1}\left\|G_{1}(z)\right\|_{\mathfrak{Q}\left(u_{1}, y_{1}\right)} & \text { in the discrete-time case. }\end{cases}
$$

Consequently, the $\mathrm{H}^{\infty}$-optimal control problem can be formulated as

$$
\underset{\Sigma_{\mathrm{K}} \text { stabilizing }}{\operatorname{minimize}}\left\|G_{1}\right\|_{\infty} \text {. }
$$

Notice that (2.2) is equivalent to the disturbance-attenuation problem, since the $\mathrm{H}^{\infty}$-norm of $G_{1}$ is precisely equal to the norm of the bounded linear map from $u_{1}(\cdot) \in \mathbf{L}_{2}\left(\mathbb{R}_{+}, \mathcal{U}_{1}\right)$ to $y_{1}(\cdot) \in \mathrm{L}_{2}\left(\mathbb{R}_{+}, \mathcal{Y}_{1}\right)$ defined for zero initial conditions, where $\mathbb{R}_{+}=[0, \infty)$.

When we have access to the system state, i.e. $C_{2}=I$ and $D_{21}=0$, we deal with the state-feedback $\mathrm{H}^{\infty}$ optimal-control problem. When the system state is not available in the controller design process, we shall use the term output-feedback $\mathrm{H}^{\infty}$ optimalcontrol problem. In both cases we shall focus on the so called $\mathrm{H}^{\infty}$ suboptimal-control problem: designing $\mathrm{K}$ such that

$$
\left\|G_{1}\right\|_{\infty}<\gamma
$$

where $\gamma>0$ is a prespecified bound. Without loosing generality, we shall assume that

$$
\gamma=1 \text {. }
$$

This can be done by an appropriate scaling of the operators involved in the expressions of the transfer functions $G_{i j}(i, j=1,2)$; see [19: pp. 178]. As is well known from the literature, the solution to the $\mathrm{H}^{x}$ control problem represents the input of the $\gamma$-iteration procedure which gives, in the limiting case, the exact solution to the $\mathrm{H}^{x}$ optimal-control problem. Since we shall not be concerned with the optimal case, we shall refer to the suboptimal case simply as to the $\mathbf{H}^{x}$ optimal-control problem.

In this paper, we shall give a digital solution to the $\mathrm{H}^{x}$ control problem for the Pritchard-Salamon class of infinite-dimensional systems with unbounded input and output operators. The solution to the digital control problem is obtained by applying the discrete Popov theory to the equivalent discrete-time system obtained by lifting the hybrid $T$-periodic Pritchard-Salamon digital control system.

1.1.2 The standard digital control configuration. We have formulated the $\mathrm{H}^{x}$ control problem, both in continuous time and in discrete time. The ultimate goal is to obtain a digital solution to the aforementioned control problem considered in continuous time. Therefore we shall restrict ourselves to controllers of the form depicted in Fig. 4, where A-D and D-A represent the 'analog-to-discrete' and 'discrete-toanalog' devices, assumed to be synchronized in time and with a given sampling period $T>0$. 


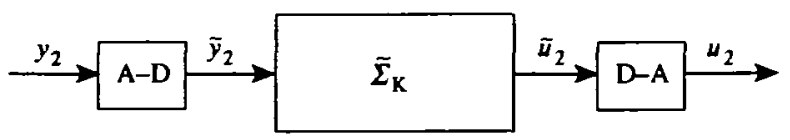

Fig. 4. The digital controller.

The designable element is now the discrete-time controller $\tilde{\Sigma}_{\mathrm{K}}$, and the closed-loop system is as in Fig. 5. Here $\mathrm{S}_{T}$ and $\mathrm{H}_{T}$ denote the sampler and the zeroth-order hold operators respectively, which will be rigorously introduced later on. A few remarks should be made from the very beginning about the set-up depicted in Fig. 5 .

(i) We deal with a hybrid (closed-loop) system since the state of the plant $\Sigma_{\mathrm{G}}$ evolves in continuous time and the state of the controller $\Sigma_{\mathrm{K}}$ in discrete time.

(ii) The closed-loop system from $u_{1}$ to $y_{1}$ is no longer time-invariant, due to the periodic characteristic of the A-D and D-A devices. Therefore, a transfer function from $u_{1}$ to $y_{1}$ cannot be defined.

(iii) Certain assumptions should be clearly made on the initial data. For example, we cannot allow a direct feedthrough from the disturbance input to the controlled output. This is especially true since, when giving a mathematical model of the A-D device, we deal with the sample operator which is not well defined on $L_{2}\left(\mathbb{R}_{+}, \mathcal{U}_{1}\right)$, the space of the disturbance input.

The main technique we use to overcome those difficulties is the lifting technique. The idea of lifting is to break up a signal defined on the real line into the sequence of signals given by the restriction of the original continuous-time signal to time intervals of the form $[k T,(k+1) T)$, where $T>0$ is the fixed sampling step and $k$ is in the set of integers. Such a lifting technique applied to the hybrid control system leads to a discrete-time representation for which the original digital control problem is converted into a discrete-time control problem. We show that a solution to the latter is also a solution to the former.

\subsection{Structure of this study}

The structure of the paper is the following. Since the infinite-dimensional systems considered in this paper present unboundedness in control and/or observation, it is of crucial importance to begin with defining the type of unboundedness which is considered. This is done in Section 2, where we introduce the Pritchard-Salamon

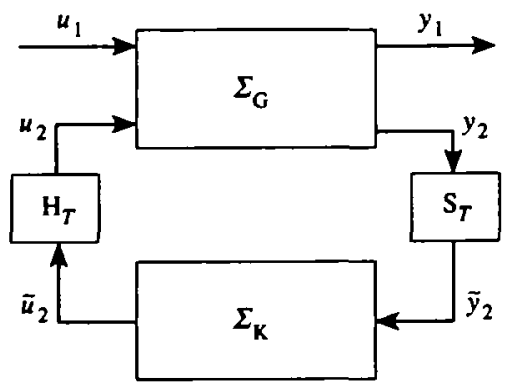

Fig. 5. The standard digital control configuration. 
class as well as the concept of Pritchard-Salamon-Popov triples in the spirit of [28, 37]. Also in Section 2, we formulate in its full generality the digital optimal control problem for Pritchard-Salamon-Popov triples. This problem is shown to be sufficiently general to include as particular cases the digital LQ optimal-control problem for Pritchard-Salamon systems and the digital $\mathbf{H}^{x}$ control problem.

Section 3 is devoted to the so-called discrete-time Popov theory. In the main result, the existence of a stabilizing solution to the discrete-time Riccati equation on a real separable Hilbert space is related to the invertibility of a certain Toeplitz operator associated with the discrete Popov triple, which is the basic object that this theory operates upon. Several applications of this discrete-time Riccati-equation theory are presented, such as discrete-time $L Q$ optimal control and discrete-time $\mathrm{H}^{\infty}$ control. For a more extended and complete treatment of these latter subjects, the reader is referred to the book [19].

Section 4 is entirely devoted to the digital stability of Pritchard-Salamon systems. The main result on exponential stability of Pritchard-Salamon systems under digital state feedback and various consequences of this result are given in Section 4.1. Since the Popov theory requires exponential/power stability of the system, we shall assume it from the very beginning. Since apparently it is a strong assumption, we shall tackle the question of how it can be removed within the framework of Popov theory without paying a too high price. Another thing we accomplish in this section is the full generalization to Pritchard-Salamon systems of the finite-dimensional results on so-called hybrid stability from [11]. Two subsections are devoted to the lifting technique. The results on lifting outlined here represent the minimum background for the reader to understand how this digital control tool operates and how to apply such a technique to reduce the digital control problem to an equivalent discretetime one.

In Section 5 we give the main digital control results of this paper. Our attention is focused on the following control problems for systems in the Pritchard-Salamon class: (i) the digital LQ optimal control problem and (ii) the digital $\mathrm{H}^{x}$ control problem. We show how these digital control problems can be converted into equivalent discrete-time control problems. The main results are given by applying the discrete Popov-theory result outlined in Section 3.

In Section 6 we reach the conclusions of this paper and we summarize the main investigation directions and interesting open problems that arose during this study.

\section{Motivation. Systems with unboundedness in control and observation}

The aim of this section is to present to the reader the difficulties arising when solving the digital control problem for systems with certain unboundedness in control and observation. We consider first the digital control problem for linear time-invariant infinite-dimensional systems with bounded input operators.

Definition 2.1 Let $\mathcal{X}$ and $\mathcal{U}$ be real separable Hilbert spaces. A triple of the form

$$
\Sigma\left(S(\cdot) . B . M=\left[\begin{array}{cc}
Q & L \\
L^{*} & R
\end{array}\right]=M^{*}\right)
$$


with $S(\cdot)$ a strongly continuous semigroup of bounded operators on $\mathcal{X}, B \in \mathfrak{Q}(\mathcal{U}, \mathcal{X})$, $Q=Q^{*} \in \mathfrak{I}(\mathcal{X}), L \in \mathfrak{I}(\mathcal{X}, \mathcal{U})$, and $R=R^{*} \in \mathfrak{L}(\mathcal{U})$, is called a Popov triple on $(\mathcal{X}, \mathcal{U})$.

We associated with the Popov triple $\Sigma(S(\cdot), B, M)$ the following objects:

(i) the initial-value problem

$$
\dot{x}(t)=A x(t)+B u(t), \quad x(0)=x_{0} \in \mathcal{X},
$$

where $A$ is the infinitesimal generator of $S(\cdot)$ : a strongly continuous semigroup of bounded operators on $\mathcal{X}$;

(ii) the quadratic index

$$
J_{\Sigma}\left(x_{0}, u(\cdot)\right) \triangleq \int_{0}^{\infty}\left\langle\left[\begin{array}{cc}
Q & L \\
L^{*} & R
\end{array}\right]\left[\begin{array}{l}
x(t) \\
u(t)
\end{array}\right],\left[\begin{array}{l}
x(t) \\
u(t)
\end{array}\right]\right\rangle_{\mathcal{X} \times u} \mathrm{~d} t
$$

where $x(\cdot)$ is the mild solution to the initial-value problem (2.3).

(iii) the class of admissible control functions

$$
\mathcal{U}_{\mathrm{adm}}^{x_{0}} \triangleq\left\{u(\cdot) \in \mathrm{L}_{2}\left(\mathbb{R}_{+}, \mathcal{U}\right): x(\cdot)=S(\cdot) x_{0}+\int_{0}^{\cdot} S(\cdot-\tau) B u(\tau) \mathrm{d} \tau \in \mathrm{L}_{2}\left(\mathbb{R}_{+}, \mathcal{X}\right)\right\}
$$

where $\mathcal{U}$ is the space of controls.

REMARK 2.2 Proposition 3.10 from [37] gives the set of necessary and sufficient conditions for which $\mathcal{U}_{\mathrm{adm}}^{x_{0}}$ is not void. To be more precise, assume that $(S(\cdot), B)$ is stabilizable, and let $F \in \mathfrak{Q}(\mathcal{X}, \mathcal{U})$ be any stabilizing feedback operator. Then $u(\cdot) \in \mathcal{U}_{\mathrm{adm}}^{x_{0}}$ if and only if there exists $v(\cdot) \in \mathrm{L}_{2}\left(\mathbb{R}_{+}, \mathcal{U}\right)$ such that

$$
u(\cdot)=F x(\cdot)+v(\cdot) \text {. }
$$

Let $\mathrm{S}(\mathbf{N}, \mathcal{Z})$ and

$$
\mathrm{PC}_{T}\left(\mathbb{R}_{+} ; \mathcal{Z}\right) \triangleq\left\{z(\cdot): \mathbb{R}_{+} \rightarrow \mathcal{Z}: z(t)=z(k T)(k T \leqslant t<k T+T) \text { for } k \in \mathbb{N}\right\}
$$

denote the spaces of $\mathcal{Z}$-valued sequences and $\mathcal{Z}$-valued $T$-interval piecewise constant functions respectively, where $\mathbf{N}=\{0,1, \ldots\}$. Let $\mathrm{C}\left(\mathbb{R}_{+}, \mathcal{Z}\right)$ be the space of continuous $\mathcal{Z}$-valued functions on $\mathbb{R}_{+}$. Define the sample and zeroth-order hold operators of period $T$ by

$$
\begin{aligned}
& \mathrm{S}_{T}: \mathrm{C}\left(\mathbb{R}_{+}, \mathcal{Z}\right) \rightarrow \mathrm{S}(\mathbf{N}, \mathcal{Z}), \quad \mathrm{S}_{T} \approx \triangleq(z(k T): k \in \mathbb{N}) \triangleq \tilde{z} \\
& \mathrm{H}_{T}: \mathrm{S}(\mathbf{N}, \mathcal{Z}) \rightarrow \mathrm{PC}_{T}\left(\mathbb{R}_{+}, \mathcal{Z}\right), \quad \text { zstep } \triangleq \mathrm{H}_{T} \tilde{z},
\end{aligned}
$$

such that

$$
z_{\text {step }}(t)=z(k T) \quad(k T \leqslant t<k T+T ; k \in \mathbb{N}) .
$$

REMARK 2.3 Clearly

$$
\mathrm{PC}_{T}\left(\mathbb{R}_{+}, \mathcal{Z}\right)=\mathrm{H}_{\mathcal{T}} \mathrm{S}(\mathbf{N}, \mathcal{Z}) \subset \mathrm{L}_{\epsilon}\left(\mathbb{R}_{+}, \mathcal{Z}\right),
$$

and we also have the natural isomorphism

$$
\mathrm{S}(\mathbb{N}, \mathcal{Z}) \cong \mathrm{PC}_{\mathcal{T}}\left(\mathbb{R}_{+}, \mathcal{Z}\right) \text {, }
$$


Definition 2.4 (i) Let $\tilde{u}=(u(0), u(1), \ldots)$ denote a sequence in $\mathrm{S}(\mathbf{N} ; \mathcal{U})$. A piecewise constant control function $u_{\text {step }}(\cdot)$, defined by

$$
u_{\text {step }}(t)=\left(\mathbf{H}_{T} \tilde{u}\right)(t) \quad\left(t \in \mathbb{R}_{+}\right) \text {, }
$$

is called a digital exponential stabilizing control for the initial-value problem (2.3) if, for $u(\cdot)=u_{\text {step }}(\cdot)$, the solution to (2.3) decays exponentially to zero as $t$ tends to infinity, i.e.

$$
\|x(t)\|_{\mathcal{X}} \leqslant \mu \mathrm{e}^{-\alpha t}\left\|x_{0}\right\|_{\mathcal{X}} \quad\left(t \in \mathbb{R}_{+}\right)
$$

for some constants $\mu>1$ and $\alpha>0$.

(ii) A pair $(S(\cdot), B)$ is called digitally exponentially stabilizable if there exists a digital control law in feedback form, i.e. if there exists a feedback operator $F \in \mathfrak{L}(\mathcal{X}, \mathcal{U})$ such that, for the control function of the form

$$
u_{\text {step }}(t)=F x(k T) \quad(k T \leqslant t<k T+T),
$$

the solution to the initial-value problem decays exponentially to zero as $t$ tends to infinity.

The digital optimal control problem for infinite-dimensional systems with bounded input and bounded output operators can be formulated as

$$
\underset{u \in \mathcal{U}_{\text {sdm }}^{\mathrm{r}_{0} \cap \mathrm{PC}_{T}\left(\mathbf{R}_{+}, \mathcal{u}\right)}}{\operatorname{minimize}} J_{\Sigma}\left(x_{0}, u_{\text {step }}(\cdot)\right) \text {. }
$$

As is well known (see Lee et al. [25], Hager \& Horowitz [17], or the recent paper of Rosen \& Wang [29]), under certain circumstances the solution to this problem can be expressed in the state-feedback form, i.e. there exists a feedback gain operator $F \in \mathfrak{I}(\mathcal{X}, \mathcal{U})$ such that

$$
u_{\text {step }}^{\text {opt }}(\cdot)=F x\left(\cdot, x_{0}, u_{\text {step }}^{\text {opt }}(\cdot)\right) \text { for all } x_{0} \in \mathcal{X}
$$

or, expressed alternatively,

$$
u_{\text {step }}^{\text {opt }}=\mathrm{H}_{T} F \tilde{x},
$$

where $u_{\text {step }}^{\text {opt }}(\cdot)$ is the exponentially stabilizing control-law input that achieves the minimum in (2.4).

\subsection{Pritchard-Salamon sy'stems}

As is well known, most of the interesting finite-dimensional control systems encountered in practice do not fall in the framework of systems with bounded input and output operators. For systems formally described by

$$
\begin{aligned}
& i(t)=A x(t)+B u(t), \quad x(0)=x_{0}, \\
& r(t)=C x(t)+D u(t),
\end{aligned}
$$

the unboundedness of the $B$ and $C$ operators is usually considered in the sense of admissibility. One special class of infinite-dimensional systems with unbounded $B$ and $C$ operators has recently captured the attention of specialists due to its nice 
structural properties, especially in relation to the linear quadratic control problem. It is the so called Pritchard-Salamon class of systems (see Pritchard \& Salamon [28]).

DefinItION 2.5 Let $\mathcal{W}, \mathcal{X}, \mathcal{V}, \mathcal{U}, \mathcal{Y}$ be real separable Hilbert spaces admitting the continuous dense injections

$$
W \hookrightarrow \mathcal{X} \hookrightarrow \mathcal{V} .
$$

Let $S^{\mathcal{V}}(\cdot)$ be a $\mathrm{C}_{0}$ semigroup with the infinitesimal generator $A^{\mathcal{V}}$ on $\mathcal{V}$ which restricts to $\mathrm{C}_{0}$ semigroups $S^{\mathcal{X}}(\cdot)$ and $S^{\mathcal{W}}(\cdot)$ with infinitesimal generators $A^{\mathcal{X}}$ and $A^{\mathcal{W}}$ on $\mathcal{X}$ and $\mathcal{W}$ respectively.

(i) An operator $B \in \mathfrak{L}(\mathcal{U}, \mathcal{V})$ is called an admissible input operator for $S(\cdot)$ with respect to $(\mathcal{W}, \mathcal{V})$, if there exists some $t>0$ and $c>0$ such that

$$
\int_{0}^{t} S(t-\tau) B u(\tau) \mathrm{d} \tau \in W, \quad\left\|\int_{0}^{t} S(t-\tau) B u(\tau) \mathrm{d} \tau\right\|_{\mathcal{W}} \leqslant c\|u(\cdot)\|_{\mathrm{L}_{2}(0, t ; u)}
$$

for all $u(\cdot) \in \mathrm{L}_{2}\left(\mathbb{R}_{+}, \mathcal{U}\right)$.

(ii) An operator $C \in \mathfrak{S}(\mathcal{W}, \mathcal{Y})$ is called an admissible output operator for $S(\cdot)$ with respect to $(\mathcal{W}, \mathcal{V})$, if there exists some $t>0$ and $c>0$ such that

for all $x \in \mathcal{W}$.

$$
\|C S(\cdot) x\|_{\mathrm{L}_{2}(0, t ; y)} \leqslant c\|x\|_{\mathcal{V}}
$$

Remark 2.6 The admissibility of $C$ implies that the linear map from $W$ to $\mathrm{L}_{2}\left(\mathbb{R}_{+}, \mathcal{Y}\right)$ defined by $x \mapsto C S(\cdot) x$ has a unique bounded extension from $\mathcal{V}$ to $\mathrm{L}_{2}\left(\mathbb{R}_{+}, \mathcal{Y}\right)$. We shall denote it by $x \mapsto \overline{C S}(\cdot) x(x \in \mathcal{V})$.

Definition 2.7 Let $B \in \mathfrak{L}(\mathcal{U}, \mathcal{V})$ and $C \in \mathfrak{L}(\mathcal{W}, \mathcal{Y})$ be admissible input and output operators for $S^{\mathcal{V}}(\cdot)$ with respect to $(\mathcal{W}, \mathcal{V})$, and suppose that $D \in \mathfrak{L}(\mathcal{U}, \mathcal{Y})$. Then the linear infinite-dimensional system given by

$$
\begin{aligned}
& x(t)=S^{\mathcal{\nu}}(t) x_{0}+\int_{0}^{t} S^{\mathcal{\nu}}(t-\tau) B u(\tau) \mathrm{d} \tau \\
& y(\cdot)=\overline{C S}(\cdot) x_{0}+C \int_{0}^{\cdot} S(\cdot-\tau) B u(\tau) \mathrm{d} \tau+D u(\cdot),
\end{aligned}
$$

where $x_{0} \in \mathcal{V}$ and $u \in \mathrm{L}_{2}^{\text {loc }}\left(\mathbb{R}_{+}, \mathcal{U}\right)$ is called a Pritchard-Salamon system and is denoted by $\Sigma(S(\cdot), B, C, D)$. If, in addition, we have

$$
\mathrm{D}\left(A^{\mathcal{V}}\right) \hookrightarrow \mathcal{W}
$$

then we call the system a smooth Pritchard-Salamon system.

Remark 2.8 (Properties of Pritchard-Salamon systems) Hypotheses (2.5a,b) implies that, for every $x_{0} \in \mathcal{W}$ and every $u(\cdot) \in \mathrm{L}_{2}(0, t ; \mathcal{U})$, formula (2.7a) defines a continuous function $x(\cdot)$ on the interval $(0, t)$ with values in $\mathcal{W}$. The output function can be defined by

$$
y(t)=C S^{\mathcal{W}}(t) x_{0}+C \int_{0}^{t} S(t-\tau) B u(\tau) \mathrm{d} \tau
$$

and is a continuous function on the interval $(0, t)$ with values in $\mathcal{Y}$. 
If $x_{0} \in \mathcal{V} \backslash \mathcal{W}$, then $x(\cdot)$ is constrained only to be a continuous function with values in $\mathcal{V}$, and (2.9) does not make sense directly. But, if the hypothesis (2.6) is satisfied, then (2.9) specifies a well-defined $\mathrm{L}_{2}$ function with values in $\mathcal{Y}$.

\subsection{Pritchard-Salamon-Popov triples}

We shall formulate firstly the digital control problem for Pritchard-Salamon systems in its full generality, in the same spirit as we did in the bounded case at the beginning of this section; after that, we shall explain why the problem is difficult and interesting, and what can be done to surmount those difficulties. The Popov triple and associated concepts have been generalized for Pritchard-Salamon systems by $\mathbf{M}$. Weiss [37].

Definition 2.9 Let $\mathcal{W}, \mathcal{V}$, and $\mathcal{U}$ be real separable Hilbert spaces, and suppose that $\mathcal{W}$ is contained in $\mathcal{V}$ with continuous dense injection $(\mathcal{W} \hookrightarrow \mathcal{V})$. Let $S^{\mathcal{V}}(\cdot)$ be a $\mathrm{C}_{0}$ semigroup, with the infinitesimal generator $A^{\mathcal{V}}$ on $\mathcal{V}$, which restricts to a $\mathrm{C}_{0}$ semigroup $S^{\mathcal{W}}(\cdot)$, with infinitesimal generator $A^{\mathcal{W}}$, on $\mathcal{W}$. Let $B \in \mathfrak{Q}(\mathcal{U}, \mathcal{V})$ be an admissible input operator for $S^{\mathcal{V}}(\cdot)$ with respect to $(\mathcal{W}, \mathcal{V})$. Then $Q=Q^{*} \in \mathfrak{L}(\mathcal{W})$ is an admissible weighting operator for $S^{\mathcal{V}}(\cdot)$ with respect to $(\mathcal{W}, \mathcal{V})$ if it is an admissible output operator and there exists $\mu>0$ and $t$ such that

$$
\int_{0}^{t}\left|\left\langle Q S^{\mathcal{W}}(\tau) x, S^{\mathcal{W}}(\tau) y\right\rangle_{\mathcal{W}}\right| \mathrm{d} \tau \leqslant \mu\|x\|_{\mathcal{V}}\left\|_{g}\right\|_{\mathcal{V}}
$$

for every $x, y \in \mathcal{W}$. A triple of the form

$$
\Sigma\left(S^{\mathcal{V}}(\cdot), B, M=\left[\begin{array}{ll}
Q & L \\
L^{*} & R
\end{array}\right]=M^{*}\right),
$$

with $L$ bounded in such a way that $L^{*} \in \mathfrak{L}(\mathcal{W}, \mathcal{U})$ is an admissible output operator for $S^{\mathcal{V}}(\cdot)$ with respect to $(\mathcal{W}, \mathcal{V})$, and with $R=R^{*} \in \mathfrak{L}(\mathcal{U})$, is called a PritchardSalamon-Popov triple on $(\mathcal{W} \hookrightarrow \mathcal{V}, \mathcal{U})$. The smallest $\mu$ satisfying $(2.10)$ is known as the Popov index.

In Figure 6 we have represented the mappings generated by a Pritchard-SalamonPopov triple. A Pritchard-Salamon-Popov triple on $(\mathcal{W} \hookrightarrow \mathcal{V}, \mathcal{U})$ is called smooth if condition (2.8) is satisfied, and regular if the operator $R$ is boundedly invertible.

Definition 2.10 Let $\Sigma(S(\cdot), B, C, D)$ be a Pritchard-Salamon system.

(i) The pair $(S(\cdot), B)$ is called admissibly (boundedly') stabilizable if there exists an admissible output operator $F \in \mathfrak{L}(\mathcal{W}, \mathcal{U})$ (an operator $F \in \mathfrak{L}(\mathcal{V}, \mathcal{U})$ ) such that the perturbed $C^{0}$ semigroup $S_{F}^{\mathrm{R}}(\cdot)$, generated by $A^{\mathcal{V}}+B F$, is exponentially stable on $\mathcal{W}$ and $\mathcal{V}$.

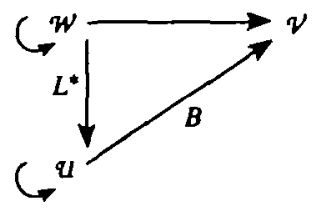

Fig. 6. The mappings generated by a Pritchard-Salamon-Popov triple. 
(ii) The pair $(C, S(\cdot))$ is called admissibly (boundedly) detectable if there exists an admissible input operator $H \in \mathfrak{Q}(\mathcal{Y}, \mathcal{V})$ (an input operator $H \in \mathfrak{Q}(\mathcal{Y}, \mathcal{W})$ ) such that the perturbed $\mathrm{C}^{0}$ semigroup $S_{H}^{\mathrm{L}}(\cdot)$, generated by $A^{\mathcal{V}}+H C$, is exponentially stable on $\mathcal{W}$ and $\mathcal{V}$.

The following result [14] gives the relationship between the two concepts of stabilizability quoted above.

Proposition 2.11 (i) The pair $(S(\cdot), B)$ is admissibly stabilizable if and only if is boundedly stabilizable. (ii) The pair $(C, S(\cdot))$ is admissibly detectable if and only if is boundedly detectable.

Let us assume that $\left(S^{\mathcal{V}}(\cdot), B\right)$ is admissibly (boundedly) stabilizable. As in the bounded case, we shall associate with the Pritchard-Salamon-Popov triple an initial-value problem and a quadratic index. The first one is easy to define. It is given by

$$
\dot{x}(t)=A^{\mathcal{V}} x(t)+B u(t), \quad x(0)=x_{0} \in \mathcal{V} .
$$

The set of admissible control functions is now

$\mathcal{U}_{\mathrm{adm}}^{x_{0}} \triangleq\left\{u(\cdot) \in \mathrm{L}_{2}\left(\mathbb{R}_{+}, \mathcal{U}\right): x(\cdot)=S^{\mathcal{V}}(\cdot) x_{0}+\int_{0}^{\bullet} S^{\mathcal{\nu}}(\cdot-\tau) B u(\tau) \mathrm{d} \tau \in \mathrm{L}_{2}\left(\mathbb{R}_{+}, \mathcal{V}\right)\right\}$

Notice that if $\left(S^{\mathcal{\nu}}(\cdot), B\right)$ is admissibly stabilizable, then $U_{\mathrm{adm}}^{x_{0}} \neq \varnothing$. Hence, for any $x_{0} \in \mathcal{W}$, there exists $u(\cdot) \in \mathcal{U}_{\mathrm{adm}}^{x_{0}}$ such that the state function $x(\cdot)$ is a squareintegrable $\mathcal{W}$-valued function, and then the (infinite-time-horizon) quadratic index associated with the Pritchard-Salamon-Popov triple $\Sigma(S(\cdot), B, M)$ is well defined by the quadratic functional

$$
J_{\Sigma}\left(x_{0}, u(\cdot)\right) \triangleq \int_{0}^{\infty}\left\langle\left[\begin{array}{ll}
Q & L \\
L^{*} & R
\end{array}\right]\left[\begin{array}{l}
x(t) \\
u(t)
\end{array}\right],\left[\begin{array}{l}
x(t) \\
u(t)
\end{array}\right]\right\rangle_{\mathcal{W} \times \mathcal{U}} \mathrm{d} t
$$

where $x(\cdot)$ is the mild solution to the initial-value problem (2.12). Martin Weiss showed in [37] that $J_{\Sigma}\left(x_{0}, u(\cdot)\right)$ defined by (2.14) can be extended to a bounded quadratic functional for $x_{0} \in \mathcal{V}$ and $u(\cdot) \in \mathcal{U}_{\mathrm{adm}}^{x_{0}}$.

\subsection{Digital feedback control law}

Definition 2.12 Let $S(\cdot)$ be a $C_{0}$-semigroup on $\mathcal{W} \hookrightarrow \mathcal{V}$, let $B \in \mathfrak{L}(\mathcal{U}, \mathcal{V})$ be an admissible input operator, and consider the initial-value problem on $\mathcal{W}$ given by

$$
\dot{x}(t)=A^{\mathcal{W}} x(t)+B u(t), \quad x_{0} \in \mathcal{W},
$$

where $A^{\mathcal{W}}$ is the infinitesimal generator of $S^{\mathcal{W}}(\cdot)$. Let $F \in \mathfrak{L}(\mathcal{V}, \mathcal{U})$ be a feedback operator and let $\tilde{x}=(x(k T): k \in \mathbf{N})$ be the sampled-state sequence.

(i) A piecewise constant control function of the form

$$
u_{\text {step }}(t)=\left(\mathrm{H}_{T} \underline{F} \tilde{x}\right)(t)=F x(k T) \quad(k T \leqslant t \leqslant k T+T),
$$


is called a digital feedback control law for the pair $(S(\cdot), B)$, where $\underline{F}$ is the natural extension of $F$ to sequences in $\mathcal{V}$, namely $\underline{F} \tilde{x}=(F x(k T): k \in \mathbf{N})$.

(ii) If the solution to the initial-value problem (2.15) under the digital feedback control law decays exponentially to zero as $t$ tends to infinity, i.e.

$$
\|x(t)\|_{\mathcal{W}} \leqslant \mu_{\mathcal{W}} \mathrm{e}^{-\alpha_{w} t}\left\|x_{0}\right\|_{\mathcal{W}} \quad\left(t \in \mathbb{R}_{+}\right)
$$

for some $\mu_{\mathcal{W}}>1$ and $\alpha_{\mathcal{W}}>0$, then the digital feedback control law is called an exponentially stabilizing digital feedback.

We can formulate the digital optimal control problem for infinite-dimensional systems with unbounded input and unbounded output operators that belong to the PritchardSalamon class as follows: determine an exponentially stabilizing digital feedback $u \in \mathcal{U}_{\mathbf{a d m}}^{x_{0}} \cap \operatorname{PC}_{T}\left(\mathbb{R}_{+}, \mathcal{U}\right)$ minimizing the quadratic index $J_{\Sigma}\left(x_{0}, u_{\text {step }}(\cdot)\right)$, i.e.

$$
\underset{u \in \mathcal{U}_{\mathbf{x} \text { dm }}^{x_{0}} \cap \mathbf{P C}_{T}\left(\mathbf{R}_{+}, \mathcal{U}\right)}{\operatorname{minimize}} J_{\Sigma}\left(x_{0}, u_{\text {step }}(\cdot)\right)
$$

where $J_{\Sigma}\left(x_{0}, u_{\text {step }}(\cdot)\right)$ is defined by

$$
J_{\Sigma}\left(x_{0}, u(\cdot)\right) \triangleq\left\langle\left[\begin{array}{cc}
\hat{P}_{0} & \hat{P} \\
\hat{P}^{*} & \hat{R}
\end{array}\right]\left[\begin{array}{c}
x_{0} \\
u(\cdot)
\end{array}\right],\left[\begin{array}{c}
x_{0} \\
u(\cdot)
\end{array}\right]\right\rangle_{\mathcal{W} \times \mathrm{L}_{2}\left(\mathbf{R}_{+}, u\right)}\left(x_{0} \in \mathcal{W}\right),
$$

with

$$
\begin{aligned}
\hat{P}_{0} & \in \mathfrak{L}(\mathcal{W}), \\
\hat{P} & \in \mathfrak{L}\left(\mathrm{L}_{2}\left(\mathbb{R}_{+}, \mathcal{U}\right), \mathcal{W}\right), \\
\hat{R} & \in \mathfrak{L}\left(\mathrm{L}_{2}\left(\mathbb{R}_{+}, \mathcal{U}\right)\right) .
\end{aligned}
$$

The above formulation of the digital optimal control problem for Pritchard-Salamon systems shows clearly our intention to restrict to the case

$$
x_{0} \in \mathcal{W}
$$

and, anticipating a little, to be basically concerned mainly with stabilization on $\mathcal{W}$. However, the case when $x(t)$ evolves generally in $\mathcal{V}$ is also considered in order to give the reader as complete a picture as possible of the difficulties occurring in digital control of systems with unboundedness.

\section{Discrete-time Popov theory}

Originally developed by Ionescu \& Weiss [21] for finite-dimensional continuous-time and discrete-time systems, the main results on discrete Popov theory have been completely generalized to the case of time-varying discrete-time systems on Hilbert spaces in $[18,19]$. Among the various results of Popov theory, the one giving the link between a quadratic cost functional and the solution to the so-called KalmanSzego-Popov-Yakubovitch system, strongly related to Riccati-equation theory, is (in our opinion) the most relevant. The central result proved in $[21,18]$ was a condition equivalent to the Popov positivity condition expressed in terms of the invertibility of a certain Toeplitz-like operator. Immediate applications, probably 
the most important ones, were to write down the solution to the discrete-time LQ optimal-control and $\mathrm{H}^{\infty}$ control problems. In this section, we give the main results on discrete Popov theory from $[18,19,21]$.

\subsection{Discrete-time Popov triples. Basic concepts.}

We shall begin this section by outlining some basic definitions that are needed for the further developments.

Definition 3.1 Let $\Sigma(A, B, C, D)$ be an infinite-dimensional discrete-time system with the discrete-time operator $A \in \mathfrak{L}(\mathcal{X})$, and let $B \in \mathfrak{L}(\mathcal{U}, \mathcal{X}), C \in \mathfrak{I}(\mathcal{X}, \mathcal{Y})$, and $D \in \mathfrak{L}(\mathcal{U}, \mathcal{Y})$.

(i) The pair $(A, B)$ is called power-stabilizable on $\mathcal{X}$ if there exists $F \in \mathfrak{L}(\mathcal{X}, \mathcal{U})$ such that the discrete-time perturbed operator $A+B F$ is power-stable on $\mathcal{X}$.

(ii) The pair $(C, A)$ is called power-detectable on $\mathcal{X}$ if there exists $H \in \mathfrak{L}(\mathcal{Y}, \mathcal{X})$ such that the discrete-time perturbed operator $A+H C$ is power-stable on $\mathcal{X}$.

Definition 3.2 Let $\mathcal{X}$ and $\mathcal{U}$ be real separable Hilbert spaces. A triple of the form

$$
\Sigma\left(A, B, M=\left[\begin{array}{ll}
Q & L \\
L^{*} & R
\end{array}\right]=M^{*}\right)
$$

with $A \in \mathfrak{L}(\mathcal{X})$ the generator of a discrete-time power-stable semigroup on $\mathcal{X}$, $B \in \mathfrak{L}(\mathcal{U}, \mathcal{X}), Q=Q^{*} \in \mathfrak{L}(\mathcal{X}), L \in \mathfrak{L}(\mathcal{U}, \mathcal{X})$, and $R=R^{*} \in \mathfrak{L}(\mathcal{U})$, is called a discrete Popov triple on $(\mathcal{X}, \mathcal{U})$.

We associate with the discrete Popov triple $\Sigma(A, B, M)$ the following objects:

(i) the initial-value problem

$$
x(k+1)=A x(k)+B u(k), \quad x(0)=x_{0} \in \mathcal{X},
$$

where $A \in \mathfrak{L}(\mathcal{X})$ and $B \in \mathfrak{I}(\mathcal{U}, \mathcal{X})$;

(ii) the quadratic index

$$
J\left(x_{0}, u(\cdot)\right)=\sum_{k=0}^{\infty}\left\langle\left[\begin{array}{cc}
Q & L \\
L^{*} & R
\end{array}\right]\left[\begin{array}{l}
x(k) \\
u(k)
\end{array}\right],\left[\begin{array}{l}
x(k) \\
u(k)
\end{array}\right]\right\rangle_{\mathcal{X} \times u}
$$

(iii) the class of admissible control sequences

$$
\mathcal{U}_{\mathrm{adm}}^{x_{0}} \triangleq\left\{u(\cdot) \in \ell_{2}(\mathbf{N}, \mathcal{U}):\left(k \mapsto x(k)=A^{k} x_{0}+\sum_{i=0}^{k-1} A^{k-i-1} B u(i)\right) \in \ell_{2}(\mathbf{N}, \mathcal{X})\right\}
$$

Remark 3.3 Since $A$ has been assumed to be power-stable on $\mathcal{X}$, then

$$
\mathcal{U}_{\mathrm{adm}}^{x_{0}}=\ell_{2}(\mathbf{N}, \mathcal{U})
$$

for every $x_{0} \in \mathcal{X}$. 
Let us define the following operators:

$$
\begin{aligned}
& \Phi: \mathcal{X} \rightarrow \ell_{2}(\mathbb{N}, \mathcal{X}), \quad(\Phi x)(k)=A^{k} x \\
& \Psi: \ell_{2}(\mathbf{N}, \mathcal{U}) \rightarrow \ell_{2}(\mathbf{N}, \mathcal{X}), \quad(\Psi u(\cdot))(k)=\sum_{i=0}^{k-1} A^{k-1-1} B u(i) .
\end{aligned}
$$

Then, for every $\left(x_{0}, u(\cdot)\right) \in \mathcal{X} \times \ell_{2}(\mathbb{N}, \mathcal{U})$, the discrete-time initial-value problem (3.1) has a unique solution $x \in \ell_{2}(\mathbf{N}, \mathcal{X})$ given by

$$
x\left(x_{0}, u(\cdot)\right)=\Phi x_{0}+\Psi u(\cdot) .
$$

Definition 3.4 Let $\Sigma(A, B, M)$ be a discrete Popov triple on $(\mathcal{X}, \mathcal{U})$.

(i) The Riccati equation associated with $\Sigma(A, B, M)$ is defined to be the following equation in the unknown $X=X^{*} \in \mathfrak{L}(\mathcal{X})$ :

$$
A^{*} X A-X-\left(A^{*} X B+L\right)\left(R+B^{*} X B\right)^{-1}\left(L^{*}+B^{*} X A\right)+Q=0 .
$$

(ii) An operator $X=X^{*} \in \mathfrak{Q}(\mathcal{X})$ satisfying (3.5), for which $R+B^{*} X B$ has a bounded inverse and for which $A+B F$ is power-stable on $\mathcal{X}$, with the operator $F \in \mathfrak{Q}(\mathcal{X}, \mathcal{U})$ defined by

$$
F \triangleq-\left(R+B^{*} X B\right)^{-1}\left(L^{*}+B^{*} X A\right)
$$

is called a stabilizing solution to the discrete-time Riccati equation (3.5), and the feedback operator $F \in \mathfrak{I}(\mathcal{X}, \mathcal{U})$ defined by (3.6) is called a stabilizing feedback.

Remark 3.5 If $R+B^{*} X B$ has a bounded inverse, then a straightforward computation shows that the discrete-time Riccati equation (3.5) is equivalent to the following so-called Kalman-Szego-Popov-Yakubovitch (KSPY) system

$$
G=R+B^{*} X B, \quad H=L+A^{*} X B, \quad H G^{-1} H^{*}=Q+A^{*} X A-X .
$$

Furthermore, the discrete-time Riccati equation (3.5) has a self-adjoint stabilizing solution $X=X^{*} \geqslant 0$ if and only if the KSPY system (3.7) has a solution $(V . W, X)$ with $V$ boundedly invertible, $X=X^{*} \geqslant 0$, and $A+B V^{-1} W$ powerstable. We shall use the KSPY system instead of the Riccati equation when we write down the solution to the discrete-time $\mathrm{H}^{x}$ control problem, since it is a more convenient way to express the stabilizing feedback operator. The reader is referred for more details to Chapter 3 of [19].

The discrete quadratic functional admits an equivalent representation (see [18] for details)

where

$$
J\left(x_{0} \cdot u\right)=\left\langle\left[\begin{array}{c}
x_{0} \\
u(\cdot)
\end{array}\right] \cdot\left[\begin{array}{cc}
\tilde{P}_{0} & \tilde{P} \\
\tilde{P}^{*} & \tilde{R}
\end{array}\right]\left[\begin{array}{c}
x_{0} \\
u(\cdot)
\end{array}\right]\right\rangle_{\mathcal{X} \times t_{2}(\tilde{x} \cdot u)}
$$

$$
\begin{aligned}
& \tilde{P}_{0}: \mathcal{X} \rightarrow \mathcal{X} . \quad \tilde{P}_{0}=\Phi^{*} \underline{Q} \Phi \\
& \tilde{P}: \mathcal{X} \rightarrow \ell_{2}(\mathbb{N}, \mathcal{U}), \quad \tilde{P}=\left(\Psi^{*} \underline{Q}+\underline{L}\right) \Phi \\
& \tilde{R}: \ell_{2}(\mathbb{N}, \mathcal{U}) \rightarrow \ell_{2}(\mathbb{N}, \mathcal{U}), \quad \tilde{R}=\underline{R}+\underline{L}^{*} \Psi+\Psi^{*} \underline{L}+\Psi^{*} \underline{Q} \Psi
\end{aligned}
$$


here the underline indicates the canonical extension of the operator to the corresponding $\ell_{2}$ sequence spaces, e.g.

$$
\underline{L}: \ell_{2}(\mathbf{N}, \mathcal{U}) \rightarrow \ell_{2}(\mathbf{N}, \mathcal{X}), \quad \underline{L}(u(k): k \in \mathbf{N}) \triangleq(L u(k): k \in \mathbf{N}) .
$$

The main discrete Popov theory result of this section, representing the time-invariant counterpart of Theorem 1 from [18], can now be stated.

TheOREM 3.6 Let $\Sigma(A, B, M)$ be a Popov triple on $(\mathcal{X}, \mathcal{U})$. Then the following assertions are equivalent.

(i) $\tilde{R} \gg 0$.

(ii) The discrete-time Riccati equation (3.5) has a stabilizing solution $X$ such that $R+B^{*} X B \gg 0$ and the solution admits the following representation

$$
X=\tilde{P}_{0}-\tilde{P} \tilde{R}^{-1} \tilde{P}^{*} .
$$

(iii) The KSPY system (3.7) has a stabilizing solution $(X, V, W)$ with $X=X^{*}$, where $V$ is boundedly invertible and for which $A-B V^{-1} W$ is power-stable. Furthermore, the stabilizing feedback operator is given by $F=-V^{-1} W$.

(iv) The quadratic index (3.2) can be expressed as

$$
J\left(x_{0}, u(\cdot)\right)=\|V u+W x\|_{2}^{2}+\left\langle x_{0}, X x_{0}\right\rangle_{\mathcal{X}}
$$

and. for the stabilizing state-feedback law

$$
u=F x=-V^{-1} W
$$

it attains its minimum value $\left\langle x_{0}, X x_{0}\right\rangle_{\mathcal{X}}$.

Proof. For the proof of the above result, the reader is referred to [19].

\subsection{The discrete-time $L Q$ optimal-control problem}

An immediate consequence of Theorem 3.6 is the following result on the solution to the (linear-quadratic) optimal-control problem associated with the discrete Popov triple $\Sigma(A, B, M)$.

\section{THEOREM 3.7 Let}

$$
\Sigma\left(A, B, M=\left[\begin{array}{ll}
Q & L \\
L^{*} & R
\end{array}\right]=M^{*}\right)
$$

with $A$ assumed power-stable on $\mathcal{X}, B \in \mathfrak{L}(\mathcal{U}, \mathcal{X}), Q=Q^{*} \in \mathfrak{L}(\mathcal{X}), L \in \mathfrak{L}(\mathcal{X}, \mathcal{U})$, and $R=R^{*} \in \mathfrak{L}(\mathcal{U})$, be a discrete Popov triple on $(\mathcal{X}, \mathcal{U})$, and assume that $\tilde{R}$, defined by $(3.8 \mathrm{c})$ is coercive. Then

$$
\min _{u(\cdot) \epsilon_{\left.i_{2} \mid \mathbb{N} \cdot u\right)}} J_{\Sigma}\left(x_{0}, u(\cdot)\right)=\min _{u(\cdot) \epsilon_{i_{2}(\mathbb{N} \cdot u)}} \sum_{k=0}^{x}\left\langle\left[\begin{array}{l}
x(k) \\
u(k)
\end{array}\right],\left[\begin{array}{cc}
Q & L \\
L^{*} & R
\end{array}\right]\left[\begin{array}{l}
x(k) \\
u(k)
\end{array}\right]\right\rangle_{\mathcal{X} \times u}
$$

exists and equals $\left\langle X x_{0}, x_{0}\right\rangle$, where $X$ is a stabilizing solution to the discrete-time 
Riccati equation (3.5). The minimum is attained for the input satisfying

$$
u(\cdot)=F x\left(x_{0}, u(\cdot)\right)
$$

where $F$ is the power-stabilizing feedback operator given by (3.6).

REMARK 3.8 Formula (3.9) is another strong motivation for the Popov approach to the digital control of infinite-dimensional systems taken in this paper. It enables one to compute the stabilizing solution to the Riccati equation (3.5) and consequently the digital exponentially stabilizing feedback operator we are looking for. In a sequel to this paper, where an example of a parabolic system which belongs to the PritchardSalamon class of systems is considered, we will show that computing the inverse of a real-valued operator enables us to write down the solution to the associated digital optimal control problem.

\subsection{The discrete-time $\mathrm{H}^{\infty}$ control problem}

The discrete-time $\mathrm{H}^{\infty}$ control problem has been addressed and solved in the finitedimensional case by several authors (Stoorvogel [32]; Iglesias [20]; Ionescu \& Weiss [22]). Its extension to the general case of infinite-dimensional discrete-time systems has been recently made by Ionescu \& Halanay [19], where the discrete Popov theory has proved to be an adequate tool for obtaining a state-space solution to the problem. In this section, we are concerned with the discrete-time $\mathrm{H}^{\infty}$ control problem in its most general form. Let us clarify this statement. The basic model considered here is the infinite-dimensional discrete-time system

$$
\Sigma\left(A,\left[B_{1}, B_{2}\right],\left[\begin{array}{l}
C_{1} \\
C_{2}
\end{array}\right],\left[\begin{array}{cc}
D_{11} & D_{12} \\
D_{21} & 0
\end{array}\right]\right)
$$

satisfying the set of equations

$$
\Sigma_{\mathrm{G}}\left\{\begin{array}{l}
(\mathrm{s} x)(k)=A x(k)+B_{1} u_{1}(k)+B_{2} u_{2}(k), \\
y_{1}(k)=C_{1} x(k)+D_{11} u_{1}(k)+D_{12} u_{2}(k), \\
y_{2}(k)=C_{2} x(k)+D_{21} u_{1}(k),
\end{array}\right.
$$

for $k \in \mathbb{N}$, where $s x=x(\cdot+1), \quad A \in \mathfrak{L}(\mathcal{X}), \quad B_{1} \in \mathfrak{I}\left(\mathcal{U}_{1}, \mathcal{X}\right), \quad B_{2} \in \mathfrak{L}\left(\mathcal{U}_{2}, \mathcal{X}\right)$, $C_{1} \in \mathfrak{I}\left(\mathcal{X}, \mathcal{Y}_{1}\right), D_{11} \in \mathfrak{I}\left(\mathcal{U}_{1}, \mathcal{Y}_{1}\right), D_{12} \in \mathfrak{L}\left(\mathcal{U}_{2}, \mathcal{Y}_{1}\right)$, and $D_{21} \in \mathfrak{L}\left(\mathcal{U}_{1}, \mathcal{Y}_{2}\right)$. We consider $\mathcal{U}_{1}, \mathcal{U}_{2}, \mathcal{Y}_{1}$, and $\mathcal{Y}_{2}$ to be the real separable Hilbert spaces of the disturbance input $u_{1}(k) \in \mathcal{U}_{1}$, control input $u_{2}(k) \in \mathcal{U}_{2}$, controlled output $y_{1}(k) \in \mathcal{Y}_{1}$, and measured output $y_{2}(k) \in \mathcal{Y}_{2}$ respectively. Here $\mathcal{X}$ is a real separable Hilbert state space, and $x(t) \in \mathcal{X}$. Unlike in [32] or [22], no coercivity constraints of the form $D_{12}^{*} D_{12} \gg 0$ or $D_{21} D_{21}^{*} \gg 0$ are necessarily imposed on the plant. This general operator framework permits us to consider the limiting case

$$
D_{21}=0 \text {, }
$$

which is referred as to the singular discrete-time $\mathrm{H}^{x}$ control problem. The importance of having available a solution to the singular discrete-time $\mathrm{H}^{x}$ control problem becomes evident in Section 5.3, where we apply the discrete-time $\mathrm{H}^{x}$ control theory 
results of this section in order to obtain a solution to the digital $\mathrm{H}^{\infty}$ control problem. The only major assumption we make on the infinite-dimensional discrete-time system is:

$$
A \text { is power-stable on } \mathcal{X} \text {. }
$$

This is a requirement specific to the discrete-time Popov theory, and details on how it can be removed are discussed later on in this paper.

Associated with the system (3.12) is the controller defined by the discrete-time system

$$
\Sigma_{\mathrm{K}} \quad\left\{\begin{array}{l}
\left(\mathrm{s} x_{\mathrm{c}}\right)(t)=A_{\mathrm{c}} x_{\mathrm{c}}(t)+B_{\mathrm{c}} y_{2}(t), \quad x_{\mathrm{c}}(0) \in \mathcal{X}_{\mathrm{c}} \\
u_{2}(t)=C_{\mathrm{c}} x_{\mathrm{c}}(t)+D_{\mathrm{c}} y_{2}(t),
\end{array}\right.
$$

where $\mathcal{X}_{\mathrm{c}}$ is the real separable Hilbert controller state space, i.e. $x_{\mathrm{c}}(t) \in \mathcal{X}_{\mathrm{c}}$, and $A_{\mathrm{c}}$, $B_{\mathrm{c}}, C_{\mathrm{c}}$, and $D_{\mathrm{c}}$ are operators bounded on appropriate subspaces.

The resultant closed-loop system is given by

$$
\Sigma_{\mathrm{GK}} \quad\left\{\begin{array}{l}
\left(\mathrm{s} x_{\mathrm{R}}\right)(t)=A_{\mathrm{R}} x_{\mathrm{R}}(t)+B_{\mathrm{R}} u_{1}(t) \\
y_{1}(t)=C_{\mathrm{R}} x_{\mathrm{R}}(t)+D_{\mathrm{R}} u_{1}(t)
\end{array}\right.
$$

where

$$
\begin{aligned}
x_{\mathrm{R}} & =\left[\begin{array}{l}
x \\
x_{\mathrm{c}}
\end{array}\right], \\
A_{\mathrm{R}} & =\left[\begin{array}{cc}
A+B_{2} D_{\mathrm{c}} C_{2} & B_{2} C_{\mathrm{c}} \\
B_{\mathrm{c}} C_{2} & A_{\mathrm{c}}
\end{array}\right], \quad B_{\mathrm{R}}=\left[\begin{array}{c}
B_{1}+B_{2} D_{\mathrm{c}} D_{21} \\
B_{\mathrm{c}} D_{21}
\end{array}\right], \\
C_{\mathrm{R}} & =\left[\begin{array}{c}
C_{1}+D_{12} D_{\mathrm{c}} C_{2} \\
D_{12} C_{\mathrm{c}}
\end{array}\right], \quad D_{\mathrm{R}}=D_{11}+D_{21} D_{\mathrm{c}} D_{21} .
\end{aligned}
$$

The augmented state space $\mathcal{X}_{\mathrm{R}}=\mathcal{X} \oplus \mathcal{X}_{\mathrm{c}}$ is a real separable Hilbert space under the inner product

$$
\left\langle x_{\mathrm{R}}, x_{\mathrm{R}}\right\rangle_{\mathcal{X}_{\mathrm{R}}}=\langle x, x\rangle_{\mathcal{X}}+\left\langle x_{\mathrm{c}}, x_{\mathrm{c}}\right\rangle_{\mathcal{X}_{\mathrm{c}}}
$$

The system (3.12) can be written in an input-output fashion as

$$
\left[\begin{array}{l}
y_{1} \\
y_{2}
\end{array}\right]=\left[\begin{array}{ll}
G_{11} & G_{12} \\
G_{21} & G_{22}
\end{array}\right]\left[\begin{array}{l}
u_{1} \\
u_{2}
\end{array}\right]
$$

where $G_{i j} \in \mathfrak{L}\left(\ell_{2}\left(\mathbf{N}, \mathcal{U}_{i}\right), \ell_{2}\left(\mathbf{N}, \mathcal{Y}_{j}\right)\right)$ are the input-output operators from $u_{i}$ to $y_{j}(i, j=1,2)$. If $G_{\mathrm{c}}$ is the input-output operator associated with the controller, then the closed-loop input-output operator is expressed as the linear fractional transformation of the system (56) and the controller (3.13):

$$
G_{1}=\mathrm{F}\left(G, G_{\mathrm{c}}\right) \triangleq G_{11}+G_{12} G_{\mathrm{c}}\left(I-G_{22} G_{\mathrm{c}}\right)^{-1} G_{21} \text {. }
$$

The discrete-time $\mathrm{H}^{\infty}$ control problem consists in finding a controller (3.13) for the system (3.12) such that the following conditions are attained.

Stability: The closed-loop system (3.14) is internally stable, i.e. $A_{\mathrm{R}}$ is power stable on $\mathcal{X}_{\mathrm{R}}$. 
Attenuation: The closed-loop input-output operator is a contraction operator:

$$
\left\|G_{1}\right\|<1 \text {. }
$$

REMARK 3.9 The combination of the closed-loop interconnection of a system $\Sigma_{\mathrm{G}}$ defined in (3.12) and the controller $\Sigma_{\mathrm{K}}$ defined by (3.13) will be denoted in this section by $\Sigma_{\mathrm{GK}}$. Its input-output operator is the linear fractional transformation of the input-output operators of the system and the controller.

Remark 3.10 The discrete-time $\mathrm{H}^{\infty}$ control problem will be referred as the statefeedback discrete-time $\mathrm{H}^{\infty}$ control problem when $C_{2}=I$ and $D_{21}=0$, and as the singular discrete-time $\mathrm{H}^{\infty}$ control problem when $D_{21}=0$.

The first basic results for the development of this section are given by the following result.

TheOrem 3.11 Let $G_{12} \in\left(\ell_{2}\left(\mathbf{N}, \mathcal{U}_{1}\right), \ell_{2}\left(\mathbb{N}, \mathcal{Y}_{2}\right)\right)$ and $G_{21} \in \mathfrak{L}\left(\ell_{2}\left(\mathbf{N}, \mathcal{U}_{2}\right), \ell_{2}\left(\mathbf{N}, \mathcal{Y}_{1}\right)\right)$ be the input-output operators from $u_{1}$ to $y_{2}$ and from $u_{2}$ to $y_{1}$, respectively. Assume that $G_{12}^{*} G_{12}$ and $G_{21} G_{21}^{*}$ are coercive. If $\Sigma_{\mathrm{K}}$ defined by (3.13) is a solution to the discrete-time $\mathrm{H}^{\infty}$ control problem, then the KSPY systems

$$
\left\{\begin{array}{c}
V^{*} J V=R+B^{*} X B \\
W^{*} J V=L+A^{*} X B \\
W^{*} J W=Q+A^{*} X A-X
\end{array}\right\}, \quad\left\{\begin{array}{c}
\hat{V}^{*} \hat{J} \hat{V}=\hat{R}+C Y C^{*} \\
\hat{W}^{*} \hat{J} \hat{V}=\hat{L}+A Y C^{*} \\
\hat{W}^{*} \hat{J} \hat{W}=\hat{Q}+A Y A^{*}-Y
\end{array}\right\}
$$

where

$$
J=\left[\begin{array}{cc}
-\mathrm{I}_{u_{1}} & 0 \\
0 & \mathrm{I}_{\mathcal{U}_{2}}
\end{array}\right] \text { and } \hat{J}=\left[\begin{array}{cc}
-\mathbf{I}_{\mathcal{Y}_{1}} & 0 \\
0 & \mathrm{I}_{\mathcal{Y}_{2}}
\end{array}\right]
$$

associated with the Popov triples

$$
\Sigma_{1}\left(A,\left[B_{1}, B_{2}\right], M=M^{*}\right), \quad \Sigma_{2}\left(A^{*},\left[C_{1}^{*}, C_{2}^{*}\right], \hat{M}=\hat{M}^{*}\right)
$$

where

$$
\begin{gathered}
M \triangleq\left[\begin{array}{ll}
Q & L \\
L^{*} & R
\end{array}\right]=\left[\begin{array}{lll}
C_{1}^{*} C_{1} & C_{1}^{*} D_{11} & C_{1}^{*} D_{12} \\
D_{11}^{*} C_{1} & D_{11}^{*} D_{11}-I_{U_{1}} & D_{11}^{*} D_{12} \\
D_{12}^{*} C_{1} & D_{12}^{*} D_{11} & D_{12}^{*} D_{12}
\end{array}\right], \\
\hat{M} \triangleq\left[\begin{array}{ll}
\hat{Q} & \hat{L} \\
\hat{L}^{*} & \hat{R}
\end{array}\right]=\left[\begin{array}{lll}
B_{1} B_{1}^{*} & B_{1} D_{11}^{*} & B_{1} D_{21}^{*} \\
D_{11} B_{1}^{*} & D_{11} D_{11}^{*}-I_{y_{1}} & D_{11} D_{21}^{*} \\
D_{21} B_{1}^{*} & D_{21} D_{11}^{*} & D_{21} D_{21}^{*}
\end{array}\right],
\end{gathered}
$$

have stabilizing solutions $(X, V, W)$ and $(Y, \hat{V}, \hat{W})$, respectively. Furthermore, 
$X \geqslant 0$ and $Y \geqslant 0$, while $V$ and $\hat{V}$ have bounded inverses of the form

$$
V=\left[\begin{array}{cc}
V_{11} & 0 \\
V_{12} & V_{22}
\end{array}\right], \quad W=\left[\begin{array}{l}
W_{1} \\
W_{2}
\end{array}\right], \quad \hat{V}=\left[\begin{array}{cc}
\hat{V}_{11} & \hat{V}_{12} \\
0 & \hat{V}_{22}
\end{array}\right], \quad \hat{W}=\left[\begin{array}{l}
\hat{W}_{1} \\
W_{2}
\end{array}\right],
$$

and $A-B V^{-1} W$ and $A-\hat{W} \hat{V}^{-1} C$ are power-stable on $\mathcal{X}$, where $B=\left[B_{1}, B_{2}\right]$ and $C=\left[\begin{array}{ll}C_{1} & C_{2}\end{array}\right]$.

Proof. For the proof of Theorem 3.11, the reader is referred to [18].

REMARK 3.12 The result given by Theorem 3.11 represents a set of necessary conditions for the existence of the solution to the discrete-time $\mathrm{H}^{\infty}$ control problem.

The rest of this section will be concerned with the set of sufficient conditions for the existence of the solution to the discrete-time $\mathrm{H}^{\infty}$ control problem. We shall consider two modified systems. The first one is obtained by the change of variables

$$
\bar{u}_{1} \triangleq V_{11} u_{1}+W_{1} x, \quad \bar{y}_{1} \triangleq V_{21} u_{1}+V_{22} u_{2}+W_{2} x
$$

with $V_{11}, W_{1}, V_{21}, V_{22}$, and $W_{2}$ as in (3.18a).

REMARK 3.13 The above change of variables is a natural step, suggested by the form (3.10) of the quadratic cost function in Theorem 3.6.

Substitute

$$
u_{1}=V_{11}^{-1} \bar{u}_{1}-V_{11}^{-1} W_{1} x
$$

into (3.11), and replace $v_{1}$ by $\bar{y}_{1}$ (notice that $V_{11}$ has a bounded inverse, since $V$ has a bounded inverse). We obtain the following modified system associated with (3.12):

$$
\Sigma_{\mathrm{O}}\left\{\begin{array}{l}
(\mathrm{s} x)(k)=A_{\mathrm{O}} x(k)+B_{\mathrm{O} 1} \bar{u}_{1}(k)+B_{\mathrm{O} 2} u_{2}(k) \\
\bar{y}_{1}(k)=C_{\mathrm{O} 1} x(k)+D_{\mathrm{O} 11} \bar{u}_{1}(k)+D_{\mathrm{O} 12} u_{2}(k) \\
y_{2}(k)=C_{\mathrm{O} 2} x(k)+D_{\mathrm{O} 21} \bar{u}_{1}(k)
\end{array}\right.
$$

where

$$
\begin{aligned}
A_{\mathrm{O}} & =A-B_{1} V_{11}^{-1} W_{1}, \quad B_{\mathrm{O} 1}=B_{1} V_{11}^{-1}, \quad B_{\mathrm{O} 2}=B_{2}, \\
C_{\mathrm{O} 1} & =W_{2}-V_{21} V_{11}^{-1} W_{1}, \quad C_{\mathrm{O} 2}=C_{2}-D_{21} V_{11}^{-1} W_{1}, \\
D_{\mathrm{O} 11} & =V_{21} V_{11}^{-1}, \quad D_{\mathrm{O} 12}=V_{22}, \quad D_{\mathrm{O} 21}=D_{21} V_{11}^{-1} .
\end{aligned}
$$

The key results from the above change of variables are the following.

Proposition 3.14 [19: Propn 5.1 (p. 161); Propn 6.1 (p. 166)]

(i) The quadratic index corresponding to the discrete-time Popov triple (3.16a) associated with (3.12) can be expressed as

$$
J\left(x_{0}, u(\cdot)\right)=-\left\|u_{1}\right\|_{2}^{2}+\left\|y_{1}\right\|_{2}^{2}
$$


(ii) Assume that $G_{12}^{*} G_{12}$ is coercive, where $G_{12} \in \mathfrak{L}\left(\ell_{2}\left(\mathbf{N}, \mathcal{U}_{1}\right), \ell_{2}\left(\mathbf{N}, \mathcal{Y}_{2}\right)\right)$ is the input-output operator from $u_{1}$ to $y_{2}$. If the change of variables (3.19) is performed, then

$$
\left\|u_{1}\right\|_{2}^{2}+\left\|y_{1}\right\|_{2}^{2}=\left\|\bar{u}_{1}\right\|_{2}^{2}+\left\|\bar{y}_{1}\right\|_{2}^{2} .
$$

THEOREM 3.15 [19: Thm 2.3 (p. 150)] Let $G_{12} \in \mathfrak{L}\left(\ell_{2}\left(\mathbf{N}, \mathcal{U}_{1}\right), \ell_{2}\left(\mathbf{N}, \mathcal{Y}_{2}\right)\right)$ and $G_{21} \in \mathcal{L}\left(\ell_{2}\left(\mathbf{N}, \mathcal{U}_{2}\right), \ell_{2}\left(\mathbf{N}, \mathcal{Y}_{1}\right)\right)$ be the input-output operators from $u_{1}$ to $y_{2}$ and from $u_{2}$ to $y_{1}$ respectively. Assume that $G_{12}^{*} G_{12}$ and $G_{21} G_{21}^{*}$ are coercive. If $\Sigma_{\mathrm{K}}$ defined by (3.13) is a solution to the discrete-time $\mathrm{H}^{\infty}$ control problem for the system $\Sigma_{\mathrm{G}}$ defined by (3.12), then the KSPY system (3.15b) written for the modified system $\Sigma_{\mathrm{O}}$ defined by $(3.20)$ has a stabilizing solution denoted $\left(Y_{\mathrm{O}}, \hat{V}_{\mathrm{O}}, \hat{W}_{\mathrm{O}}\right)$ with $Y_{\mathrm{O}} \geqslant 0$.

Remark 3.16 Equality (3.21) and the results of Proposition 3.14 suggests that $\Sigma_{\mathrm{K}}$ defined by (3.13) represents a solution to the discrete-time $\mathrm{H}^{\infty}$ control problem for the system $\Sigma_{\mathrm{G}}$ defined by (3.12) if and only if it is a solution for the modified system $\Sigma_{\mathrm{O}}$ defined by (3.20).

We have seen until now that the existence of a solution to the discrete-time $\mathbf{H}^{\infty}$ control problem implies the existence of stabilizing solutions to two KSPY systems defined by (3.15a) and (3.15b), respectively. This fact implies the existence of stabilizing solutions to the first KSPY system considered above, namely the one defined by ( $3.15 \mathrm{a})$, and to another KSPY system defined by (3.15b) associated with the modified counterpart of the original infinite-dimensional discrete-time system. In [19] it is shown that those conditions are also sufficient, as stated in the following result.

TheOREM 3.17 [19: Thm 8.1 (p. 178)] Consider the infinite-dimensional discrete-time system

$$
\Sigma\left(A,\left[B_{1}, B_{2}\right],\left[\begin{array}{l}
C_{1} \\
C_{2}
\end{array}\right],\left[\begin{array}{cc}
D_{11} & D_{12} \\
D_{21} & 0
\end{array}\right]\right),
$$

and assume that the KSPY system (3.15a) written for $\Sigma_{\mathrm{G}}$ defined by (3.12) and the KSPY system (3.15b) written for the outer system $\Sigma_{\mathrm{O}}$ defined by (3.20) have stabilizing solutions. Then there exists a solution to the discrete-time $\mathrm{H}^{\infty}$ control problem.

\section{Digital stability}

In the previous section, we stated some results (e.g. Theorem 3.6 and Theorem 3.7) under the assumption that the $A$-operator of the discrete Popov triple $\Sigma(A, B, M)$ is power-stable on $\mathcal{X}$. However, for most of the examples encountered in practice, the contrary holds. It is therefore necessary to cope with the situation when the discrete-time semigroup generator $A$ is not power-stable on $\mathcal{X}$. This can be done within the Popov-theory framework on the basis of what we shall call here feedback 
invariance. Let us introduce and explain this concept. Consider first a discrete-time Popov triple $\Sigma(A, B, M)$ where the discrete-time semigroup generator $A$ is no longer assumed to be power-stable on $\mathcal{X}$. It follows that the class of admissible control sequences associated with $\Sigma(A, B, M), \mathcal{U}_{\mathrm{adm}}^{x_{0}}$, is no longer the whole $\ell_{2}(\mathbf{N}, \mathcal{U})$. It could be empty for some pairs $\left(x_{0}, u(\cdot)\right)$, but if the pair $(A, B)$ is assumed to be power-stabilizable, then $\mathcal{U}_{\mathrm{adm}}^{x_{0}} \neq \varnothing$. This fact is assured by Propn 1.1 of [19: p. 72].

Definition 4.1 [19: Defn 1.7 (p. 75)] Let $\Sigma_{1}\left(A_{1}, B_{1}, M_{1}\right)$ and $\Sigma_{2}\left(A_{2}, B_{2}, M_{2}\right)$ be two discrete-time Popov triples on $(\mathcal{X}, \mathcal{U})$. We shall say that they are equivalent if there exists $F \in \mathfrak{L}(\mathcal{X}, \mathcal{U})$ and $X=X^{*} \in \mathfrak{I}(\mathcal{X})$ such that

$$
\begin{aligned}
& A_{2}=A_{1}+B_{1} F, \quad B_{2}=B_{1}, \\
& Q_{2}=Q_{1}+L_{1} F+F^{*} L_{1}^{*}+F^{*} R_{1} F+A_{2}^{*} X A-X, \\
& L_{2}=L_{1}+F^{*} R_{1}+A_{2} X B_{1}, \quad R_{2}=R_{1}+B_{1}^{*} X B_{1} .
\end{aligned}
$$

If $X=0$, they are called $F$-equivalent.

Related to Definition 4.1 we have the following result.

Proposition 4.2 [19: Propn 1.8 (p. 75)] Let $\Sigma_{1}$ and $\Sigma_{2}$ be two equivalent Popov triples on $(\mathcal{X}, \mathcal{U})$. If, for each $x_{0} \in \mathcal{X}$, the class of admissible control sequences is nonempty, i.e. $\mathcal{U}_{\mathrm{adm}}^{x_{0}} \neq \varnothing$, then

$$
J_{\Sigma_{1}}\left(x_{0}, u\right)=J_{\Sigma_{2}}\left(x_{0}, \bar{u}\right)+\left\langle x_{0}, X x_{0}\right\rangle_{\mathcal{X}}
$$

with

$$
\bar{u} \triangleq u-F x
$$

RemarK 4.3 Proposition 4.2 hides a very important property of F-equivalent discrete-time Popov triples, namely that the solutions to the discrete-time Ricati equations (when they exist) associated with two F-equivalent discrete-time Popov triples coincide. Furthermore, Definition 4.1 gives the transformation formulae to obtain a stable F-equivalent of an arbitrary discrete-time Popov triple.

The situation looks very similar in the continuous-time case. In [37], Martin Weiss gave a Popov-function-based solution to the (continuous) LQ control problem associated with a Pritchard-Salamon-Popov triple with $\mathrm{C}_{0}$ semigroup assumed to be exponentially stable on $\mathcal{W}$ and $\mathcal{V}$. To generalize his result, this assumption was relaxed to admissible (bounded) stabilizability of the pair $\left(S^{\mathcal{V}}(\cdot), B\right)$. In the new framework, he proved that, under an admissible (bounded) stabilizing feedback $F$, the Pritchard-Salamon-Popov triple becomes a new Pritchard-Salamon-Popov triple with respect to $(\mathcal{W} \hookrightarrow \mathcal{V}, \mathcal{U})$, defined by

$$
\Sigma_{\mathrm{F}}\left(S_{\mathrm{BF}}^{\mathcal{V}}(\cdot), B, M_{\mathrm{F}}=\left[\begin{array}{lr}
Q+N^{*} F+F^{*} N+F^{*} R F & N^{*}+F^{*} R \\
N+R F & R
\end{array}\right]=M_{\mathrm{F}}^{*}\right)
$$

with $S_{\mathrm{BF}}^{\nu}(\cdot)$ the perturbed $\mathrm{C}_{0}$-semigroup generated by $A^{\nu}+B F$. Furthermore, it has 
the remarkable property that the solutions to the Riccati equations respectively associated with $\Sigma\left(S^{\mathcal{V}}(\cdot), B, M\right)$ and $\Sigma_{\mathrm{F}}\left(S_{\mathrm{BF}}^{\mathcal{V}}(\cdot), B, M_{\mathrm{F}}\right)$ coincide, and the new stabilizing optimal feedback is obtained from the old one by subtracting $F$. Such a feedback transformation therefore permits one to extend the results to PritchardSalamon-Popov triples with the admissibly (boundedly) stabilizable pair $\left(S^{\mathcal{\nu}}(\cdot), B\right)$.

REMARK 4.4 A feedback transformation of the form (4.2) achieves only the power stability (in discrete time) of the operator $A$, and the exponential stability of the strongly continuous semigroup $S(\cdot)$, when the continuous-time case is considered. Therefore we shall call it discrete-time prestabilization and continuous-time prestabilization, respectively.

As we announced in an earlier section of this paper, we shall consider separately the cases $x_{0} \in \mathcal{W}$ and $x_{0} \in \mathcal{V}$. We assume first that $x_{0} \in \mathcal{W}$. This is a sufficient condition for having a continuous output function of the Pritchard-Salamon system $\Sigma(S(\cdot), B, C, D)$. In this case, the sampled version of the output function is well defined, and hence the digital control problem associated with the standard digital control configuration depicted in Fig. 5 can be properly formulated. As we have seen earlier in this paper, the control function considered for the digital control problem is of the form

$$
u(\cdot) \in \mathrm{PC}_{T}\left(\mathbb{R}_{+}, \mathcal{U}\right) \cap \mathcal{U}_{\mathrm{adm}}^{x_{0}}
$$

Since we have imposed that the initial value belongs to the smaller space $\mathcal{W}$, we would like to characterize the solution to the initial-value problem

$$
\dot{x}(t)=A x(t)+B u(t), \quad x(0)=x_{0} \in \mathcal{W},
$$

firstly when the control is an arbitrary $L_{2}$ function and then when it satisfies the restriction (4.3). For the first case the following result was proved in [34].

Proposition 4.5 Let $\Sigma(S(\cdot), B, C, D)$ be a Pritchard-Salamon system, and suppose that $S(\cdot)$ is exponentially stable on $\mathcal{W}$ and $\mathcal{V}$. Consider

$$
x(t)=S(t) x_{0}+\int_{0}^{t} S(t-\tau) B u(\tau) \mathrm{d} \tau
$$

and suppose that $u(\cdot) \in \mathrm{L}_{2}\left(\mathbb{R}_{+}, \mathcal{U}\right)$. Then, $x(\cdot) \in \mathrm{L}_{2}\left(\mathbb{R}_{+}, \mathcal{V}\right)$ for $x_{0} \in \mathcal{V}$, and $x(\cdot) \in \mathrm{L}_{2}\left(\mathbb{R}_{+}, \mathcal{W}\right)$ for $x_{0} \in \mathcal{W}$.

Since $\mathrm{PC}_{T}\left(\mathbb{R}_{+} \mathcal{U}\right) \subset \mathrm{L}_{2}\left(\mathbb{R}_{+}, \mathcal{U}\right)$, one can conclude immediately that, if $x_{0} \in \mathcal{W}$ and $S(\cdot)$ is exponentially stable on $\mathcal{W}$, then the state function satisfies

$$
x(\cdot) \in \mathrm{L}_{2}\left(\mathbb{R}_{+}, \mathcal{W}\right) \cap \mathrm{C}\left(\mathbb{R}_{+}, \mathcal{W}\right)
$$

An immediate consequence of the above result is to show that the sample sequence of the state function is $\mathcal{W}$-valued and square-summable.

Corollary 4.6 Let $\Sigma(S(\cdot), B, C . D)$ be a Pritchard-Salamon system, and suppose that $S(\cdot)$ is exponentially stable on $\mathcal{W}$. Assume that the control function satisfies 
(4.3) and let $\tilde{x}=(x(k T): k \in \mathbf{N})$ be the sampled state function. If $x_{0} \in \mathcal{W}$, then $\tilde{x} \in \ell_{2}(\mathbf{N}, \mathcal{W})$.

By re-writing the state equation at the sampling instants as

$$
x(k T+T)=\Phi x(k T)+\Gamma u(k T),
$$

one can immediately check that the output function sequence satisfies

$$
y(k T)=\Lambda x(k T)+\theta u(k T),
$$

where the operators in (4.4) and (4.5) are defined by

$$
\begin{array}{ll}
\Phi: \mathcal{W} \rightarrow \mathcal{W}, & \Phi x \triangleq S^{\mathcal{W}}(T) x \quad(x \in \mathcal{W}), \\
\Gamma: \mathcal{U} \rightarrow \mathcal{W}, & \Gamma u \triangleq \int_{0}^{T} S^{\mathcal{V}}(\tau) B u \mathrm{~d} \tau \quad(u \in \mathcal{U}), \\
\Lambda: \mathcal{W} \rightarrow \mathcal{Y}, & \Lambda x \triangleq C x \\
\Theta: \mathcal{U} \rightarrow \mathcal{Y}, & \Theta u \triangleq D u
\end{array}
$$

where $T$ is the sampling period.

Definition 4.7 We shall call $\Sigma(\Phi, \Gamma, \Lambda, \theta)$ defined by (4.6a-d) the time-discretized Pritchard-Salamon system.

REMARK 4.8 If we replace $\mathcal{W}$ by $\mathcal{V}$ in Corollary 4.6 , then the results still hold with minor modifications of the proof, and furthermore it is possible to write down a discretized state equation of the form (4.4) with the $\Phi$ and $\Gamma$ operators defined this time by

$$
\begin{array}{ll}
\Phi: \mathcal{V} \rightarrow \mathcal{V}, & \Phi x \triangleq S^{\mathcal{V}}(T) x \quad(x \in \mathcal{V}), \\
\Gamma: \mathcal{U} \rightarrow \mathcal{V}, \quad \Gamma u \triangleq \int_{0}^{T} S^{\mathcal{V}}(\tau) B u \mathrm{~d} \tau \quad(u \in \mathcal{U}) .
\end{array}
$$

However, since the output function does not make sense pointwise for $x_{0} \in \mathcal{V}$, it is impossible to write down the difference equation corresponding to (4.5), and hence the time-discretized Pritchard-Salamon system would not be well defined.

\subsection{Digital exponential stability}

In this section, we give a characterization of the exponential stabilizability of Pritchard-Salamon systems under the assumption that the feedback control law is a digital one.

We have introduced the Pritchard-Salamon class of systems and, for systems belonging to this class, we have introduced the definition of exponential stability along with the definition of admissible (bounded) stabilizability. We have seen that, if $x_{0} \in \mathcal{W}$, then the time-discretized Pritchard-Salamon system is well defined. We would like to characterize its properties of power stability and power stabilizability in terms of the exponential stability and admissible (bounded) stabilizability of the original Pritchard-Salamon system. 
REMARK 4.9 Since our approach to the optimal control problem is a digital one, we shall assume that the space of inputs and of outputs are finite-dimensional, i.e. we have finitely many sensors and actuators: $\mathcal{U}=\mathbb{R}^{m}$ and $\mathcal{Y}=\mathbb{R}^{P}$.

The basic result on digital exponential stability is as follows.

TheOREM 4.10 Let $S(\cdot)$ be a $\mathrm{C}_{0}$ semigroup on $\mathcal{W} \hookrightarrow \mathcal{V}$, and let $B \in \mathfrak{L}(\mathcal{U}, \mathcal{V})$ be an admissible input operator. Then the following statements hold

(i) If the continuous-time $\mathrm{C}_{0}$ semigroup $S^{\mathcal{W}}(\cdot)$ is exponentially stable on $\mathcal{W}$, then $\Phi$ defined by (4.6a) is a power-stable discrete-time operator on $\mathcal{W}$.

(ii) The operator $\Gamma$ defined by (4.6b) satisfies

$$
\Gamma \in \mathfrak{L}(\mathcal{U}, \mathcal{W}) \cap \mathfrak{Q}(\mathcal{U}, \mathcal{V})
$$

(iii) Assume that $(S(\cdot), B)$ is exponentially stabilizable on $\mathcal{W}$, and assume also that $\mathcal{U}=\mathbb{R}^{m}$, where $m \geqslant 1$. Then there exists a sufficiently small sampling period $T_{0}$ such that, for every $T<T_{0}$, the pair $(\Phi, \Gamma)$ defined by $(4.6 \mathrm{a}, \mathrm{b})$ is power-stabilizable on $\mathcal{W}$.

(iv) If a certain discrete-time feedback control law

$$
u(k T)=F x(k T),
$$

where $F \in \mathfrak{L}(\mathcal{W}, \mathcal{U})$, makes the discrete-time operator $\Phi+\Gamma F$ power-stable on $\mathcal{W}$, then

$$
u_{\text {step }}(t)=F x(k T) \quad(k T \leqslant t<k T+T)
$$

is a digital exponentially stabilizing feedback control law.

Proof. For the proof the reader is referred to [2].

REMARK 4.11 (i) The claims we made in the items (i) and (iii) of Theorem 4.10 can be extended in the sense that it is possible to prove power-stability and powerstabilizability on the both spaces $\mathcal{W}$ and $\mathcal{V}$ with minor modifications of the proof and assuming that $(S(\cdot), B)$ is admissibly stabilizable.

(ii) The result of item (ii) holds also when the input space $\mathcal{U}$ is infinite-dimensional.

(iii) The situation concerning the last item of Theorem 4.10 is quite different. If, for the case $x(\cdot) \in \mathcal{W}$ considered in Theorem $4.10, F$ is bounded from $\mathcal{W}$ to $\mathcal{U}$, then, when considering the case $x(\cdot) \in \mathcal{V}$, the operator $F$ must be bounded from $\mathcal{V}$ to $\mathcal{U}$. This is especially true since $F \in \mathfrak{L}(\mathcal{V}, \mathcal{U})$ implies $F \in \mathfrak{L}(\mathcal{W}, \mathcal{U})$ (the converse does not hold). Thus, for feedback operators that are bounded from $\mathcal{W}$ to $\mathcal{U}$ but unbounded from $\mathcal{V}$ to $\mathcal{U}$, we cannot obtain a bound for $\|x(t)\|_{\mathcal{V}}\left(t \in \mathbf{R}_{+}\right)$in terms of $\|x(K t)\|_{w}(k \in \mathbf{N})$.

Remark 4.12 In Fig. 7, we have represented the overlap of the control and observation mappings with respect to the $\mathcal{W}$ space for a Pritchard-Salamon system $\Sigma\left(S^{\mathcal{V}}(\cdot), B, C, D\right)$. The mapping representation associated with the time-discretized Pritchard-Salamon system is given in Fig. 8. It is easy to see that sampling has a beneficial smoothing effect on the original system, all operators are now bounded with respect to the smaller state space $\mathcal{W}$. For the original Pritchard-Salamon 


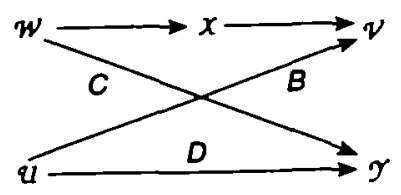

Fig. 7. The mapping representation of the Pritchard-Salamon system.

system, in general, the unboundedness of $B$ implies that the relation $B \in \mathfrak{L}(\mathcal{U} . \mathcal{W}) \cap$ $\mathfrak{S}(\mathcal{U}, \mathcal{V})$ does not hold; however, for the time-discretized Pritchard-Salamon system $\Sigma(\Phi, \Gamma, \Lambda, \theta)$, a relation corresponding to this is assured by the second item proved in Theorem 4.10 , i.e.

$$
\Gamma \in \mathfrak{Q}(\mathcal{U}, \mathcal{W}) \cap \mathfrak{Q}(\mathcal{U}, \mathcal{V})
$$

\subsection{Hybrid stability for Pritchard-Salamon systems}

The main goal of this section is to introduce the reader the concept of hybrid stability. Several properties of hybrid stable Pritchard-Salamon systems will be explored. The concept of hybrid stability was originally introduced for finite-dimensional systems in [11]. In this section, we shall extend it to Pritchard-Salamon systems. Let us consider the smooth Pritchard-Salamon system

$$
\Sigma\left(S(\cdot),\left[B_{1}, B_{2}\right],\left[\begin{array}{l}
C_{1} \\
C_{2}
\end{array}\right],\left[\begin{array}{cc}
D_{11} & D_{12} \\
0 & 0
\end{array}\right]\right)
$$

with respect to $\mathcal{W} \hookrightarrow \mathcal{V}$ and the discrete-time controller $\Sigma_{\mathrm{K}}\left(A_{\mathrm{c}}, B_{\mathrm{c}}, C_{\mathrm{c}}, D_{\mathrm{c}}\right)$ interconnected in closed loop via a sampler and a zeroth-order holder as depicted in Fig. 5.

REMARK 4.13 Notice that it is compulsory to have $D_{21}=0$. Then, for $x_{0} \in \mathcal{W}$, the solution to the measured output equation is given by

$$
y_{2}(t) \triangleq C_{2} S^{\mathcal{W}}(t) x_{0}+\int_{0}^{t} C_{2} S^{\mathcal{W}}(t-\tau)\left[B_{1} u_{1}(\tau)+B_{2} u_{2}(\tau)\right] \mathrm{d} \tau
$$

When $D_{21} \neq 0$, it follows that the solution to the measured output equation holds only in the $\mathrm{L}_{2}$ sense:

$$
y_{2}(\cdot) \triangleq C_{2} S^{\mathcal{W}}(\cdot) x_{0}+\int_{0}^{\bullet} C_{2} S^{\mathcal{W}}(\cdot-\tau)\left[B_{1} u_{1}(\tau)+B_{2} u_{2}(\tau)\right] \mathrm{d} \tau+D_{21} u_{1}(\cdot) .
$$

Hence it cannot be sampled, since the sampler is not well defined on $L_{2}$ spaces. Standard engineering practice shows that, if a low-pass filter is used prior to the sampler, then a modified sampler operator, namely the original sampler plus the

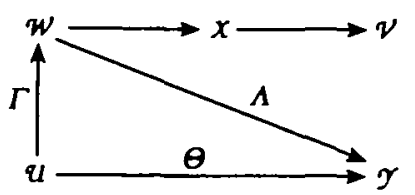

Fig. 8. The mapping representation of the time-discretized Pritchard-Salamon system. 


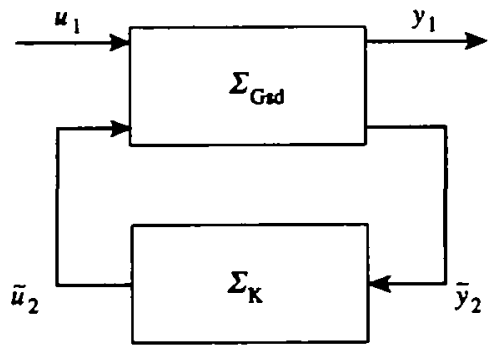

Fig. 9. The modified digital control configuration.

filter, is well defined on $\mathrm{L}_{2}$ spaces. However, since we do not want to modify the definition of the sampler, we shall assume for well-posedness that $D_{21}=0$.

Since, for a chosen sampling period $T$, the operators $\mathrm{S}_{T}$ and $\mathrm{H}_{T}$ are fixed, we shall move them around the loop and we include them into the plant obtaining the closed-loop configuration depicted in Fig. 9. The system $\Sigma_{\mathrm{Gsd}}$ is the so called sampled-data system and $\tilde{y}_{2}=\mathrm{S}_{T} y_{2}$ is the discretized measured output. By an abuse of notation, we shall denote the control sequence representing the output of the controller by $\tilde{u}_{2}$.

Let us consider

$$
G \triangleq\left[\begin{array}{ll}
G_{11} & G_{12} \\
G_{21} & G_{22}
\end{array}\right]: \mathrm{L}_{2}^{\text {loc }}\left(\mathbb{R}_{+}, \mathcal{U}_{1} \oplus \mathcal{U}_{2}\right) \rightarrow \mathrm{L}_{2}^{\text {loc }}\left(\mathbb{R}_{+}, \mathcal{Y}_{1} \oplus \mathcal{Y}_{2}\right)
$$

as an unbounded map.

It represents the input-output operator associated with the Pritchard-Salamon system (4.8):

$$
G:\left[\begin{array}{l}
u_{1} \\
u_{2}
\end{array}\right] \mapsto\left[\begin{array}{l}
y_{1} \\
y_{2}
\end{array}\right]
$$

We consider the input-output operator associated with $\Sigma_{\text {Gsd }}$, namely

$$
G_{\mathrm{sd}} \triangleq\left[\begin{array}{cc}
G_{11} & G_{12} \mathrm{H}_{T} \\
\mathrm{~S}_{T} G_{21} & \mathrm{~S}_{T} G_{22} \mathrm{H}_{T}
\end{array}\right],
$$

defined by

$$
G_{\text {sd }}: \mathrm{L}_{2}^{\text {loc }}\left(\mathbb{R}_{+}, \mathcal{U}_{1}\right) \oplus \ell_{2}^{\text {loc }}\left(\mathbf{N}, \mathcal{U}_{2}\right) \rightarrow \mathrm{L}_{2}^{\text {loc }}\left(\mathbf{R}_{+}, \mathcal{Y}_{1}\right) \oplus \ell_{2}^{\text {loc }}\left(\mathbf{N}, \mathcal{Y}_{2}\right)
$$

as an unbounded map, mapping $\left(u_{1}, \tilde{u}_{2}\right)$ to $\left(y_{1}, \bar{y}_{2}\right)$. 
Similarly to how we defined the extended closed-loop configuration in the previous section, we shall introduce two compatible exogenous signals $v \in \ell_{2}\left(\mathbf{N}, \mathcal{U}_{2}\right)$ and $w \in \ell_{2}\left(\mathbf{N} ; \mathcal{Y}_{2}\right)$ and add them to the closed-loop system. We obtain the extended digital control configuration depicted in Fig. 10.

Let us consider the mapping

$$
\left[\begin{array}{c}
u_{1} \\
v \\
w
\end{array}\right] \mapsto\left[\begin{array}{l}
y_{1} \\
\tilde{u}_{2} \\
\tilde{y}_{2}
\end{array}\right]
$$

from $\mathrm{L}_{2}^{\text {loc }}\left(\mathbb{R}_{+}, \mathcal{U}_{1}\right) \oplus \ell_{2}^{\text {loc }}\left(\mathbf{N}, \mathcal{U}_{2} \oplus \mathcal{Y}_{2}\right)$ to $L_{2}^{\text {loc }}\left(\mathbb{R}_{+}, \mathcal{Y}_{1}\right) \oplus \ell_{2}^{\text {loc }}\left(\mathbf{N}, \mathcal{U}_{2} \oplus \mathcal{Y}_{2}\right)$ described by the equation

$$
\left[\begin{array}{ccc}
I & -G_{12} \mathrm{H}_{T} & 0 \\
0 & I & -G_{\mathrm{c}} \\
0 & -\mathrm{S}_{T} G_{22} \mathrm{H}_{T} & I
\end{array}\right]\left[\begin{array}{l}
y_{1} \\
\tilde{u}_{2} \\
\tilde{y}_{2}
\end{array}\right]=\left[\begin{array}{ccc}
G_{11} & 0 & 0 \\
0 & I & 0 \\
\mathrm{~S}_{T} G_{21} & 0 & I
\end{array}\right]\left[\begin{array}{c}
u_{1} \\
v \\
w
\end{array}\right] .
$$

DefinITION 4.14 The digital control closed loop system $\Sigma_{\mathrm{GsdK}}$ is called hybrid-stable if the operator

$$
\left[\begin{array}{ccc}
I & -G_{12} \mathrm{H}_{T} & 0 \\
0 & I & -G_{\mathrm{c}} \\
0 & -\mathrm{S}_{\mathrm{T}} G_{22} \mathrm{H}_{T} & I
\end{array}\right]^{-1}\left[\begin{array}{ccc}
G_{11} & 0 & 0 \\
0 & I & 0 \\
\mathrm{~S}_{T} G_{21} & 0 & I
\end{array}\right]
$$

is well defined and bounded from

$$
\mathrm{L}_{2}\left(\mathbb{R}_{+}, \mathcal{U}_{1}\right) \oplus \ell_{2}\left(\mathbf{N}, \mathcal{U}_{2} \oplus \mathcal{Y}_{2}\right) \text { to } \mathrm{L}_{2}\left(\mathbb{R}_{+}, \mathcal{U}_{1}\right) \oplus \ell_{2}\left(\mathbf{N}, \mathcal{U}_{2} \oplus \mathcal{Y}_{2}\right),
$$

where the first factor is boundedly invertible for a given sampling step $T>0$.

Definition 4.15 Let $\Sigma_{\mathrm{G}}$ defined by (4.8) be a smooth Pritchard-Salamon system with respect to $\mathcal{W} \hookrightarrow \mathcal{X} \hookrightarrow \mathcal{V}$. We shall say that it is hybrid-stabilizable if there exists a controller $\Sigma_{\mathrm{K}}$ such that the digital control system $\Sigma_{\mathrm{GsdK}}$ is hybrid-stable.

RemarK 4.16 Assume that $I-\mathrm{S}_{T} G_{22} \mathrm{H}_{T} G_{\mathrm{c}}$ is boundedly invertible and let

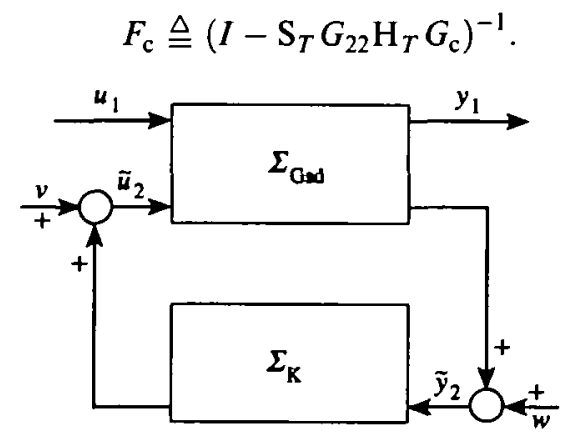

Fig. 10. The extended digital control configuration. 
Then the input-output operator associated with the extended digital closed-loop configuration is defined by

$$
\left[\begin{array}{c}
y_{1} \\
\tilde{u}_{2} \\
\tilde{y}_{2}
\end{array}\right] \triangleq G_{\mathrm{sd}}^{e}\left[\begin{array}{c}
u_{1} \\
v \\
w^{\prime}
\end{array}\right]
$$

where

$$
G_{\mathrm{sd}}^{\mathrm{e}} \triangleq\left[\begin{array}{ccc}
G_{11}+G_{12} \mathrm{H}_{T} G_{\mathrm{c}} F_{\mathrm{c}} \mathrm{S}_{T} G_{21} & G_{12} \mathrm{H}_{T} G_{\mathrm{c}} F_{\mathrm{c}} \mathrm{S}_{T} G_{22} \mathrm{H}_{T}+I & G_{12} \mathrm{H}_{T} G_{\mathrm{c}} F_{\mathrm{c}} \\
G_{\mathrm{c}} F_{\mathrm{c}} \mathrm{S}_{T} G_{21} & I+G_{\mathrm{c}} F_{\mathrm{c}} \mathrm{S}_{T} G_{22} \mathrm{H}_{T} & G_{\mathrm{c}} F_{\mathrm{c}} \\
F_{\mathrm{c}} \mathrm{S}_{T} G_{21} & F_{\mathrm{c}} \mathrm{S}_{T} G_{22} \mathrm{H}_{T} & F_{\mathrm{c}}
\end{array}\right] .
$$

Elementary computation shows that, for such a $T>0$ chosen as above, the digital closed-loop system input-output operator from $u_{1}(\cdot)$ to $y_{1}(\cdot)$ defined by

$$
G_{\mathrm{Rsd}} \triangleq G_{11}+G_{12} \mathrm{H}_{T} G_{\mathrm{c}}\left(I-\mathrm{S}_{T} G_{22} \mathrm{H}_{T} G_{\mathrm{c}}\right)^{-1} \mathrm{~S}_{T} G_{21}
$$

is bounded from $\mathrm{L}_{2}\left(\mathbb{R}_{+}, \mathcal{U}_{1}\right)$ to $\mathrm{L}_{2}\left(\mathbb{R}_{+}, \mathcal{Y}_{1}\right)$. The boundedness of $G_{\mathrm{Rsd}}$ represents the stability condition for the digital $H^{\infty}$ control problem. Notice hence that the concept of hybrid stability (Definition 4.14) is general enough to cover the stability requirement of the digital $\mathrm{H}^{\infty}$ control problem that we shall address and solve in the next section.

(iii) Notice finally that the time-domain properties of input-output operator $G_{\text {Red }}$ have no frequency-domain counterparts, since-due to the periodic characteristic of the sampler-the digital control system $\Sigma_{\mathrm{GsdK}}$ is no longer time-invariant.

4.2.1 Lifting the continuous-time system $\Sigma(S(\cdot), B, C, D)$. In this subsection, we give the main results on lifting a continuous-time $T$-periodic system to a discretetime time-invariant one. The idea is to rearrange the original periodic system in such a way that its periodicity is reflected by shift invariance in the new set-up. Let us begin with defining lifting for signals. The idea is to represent a continuous time signal by a sequence with values in a function space. In Fig. 11, we have represented schematically the original continuous time signal and its lifted counterpart.

Let us define first the lifting operator. Let $\mathcal{Z}$ be a Banach space, and recall that $\mathbf{L}_{2}\left(\mathbb{R}_{+}, \mathcal{Z}\right)$ is the space of square-integrable $\mathcal{Z}$-valued functions and $\ell_{2}(\mathbf{N}, \mathcal{Z})$ the space of square-summable $\mathcal{Z}$-valued sequences. Notice that $\mathrm{L}_{2}\left(\mathbb{R}_{+}, \mathcal{Z}\right)$ and $\ell_{2}(\mathbf{N} ; \mathcal{Z})$ are Hilbert spaces with respect to the norms induced by the inner products. Let $T$ be a fixed positive constant. Let

$$
\Omega: \mathrm{L}_{2}^{\text {loc }}\left(\mathbb{R}_{+}, \mathcal{Z}\right) \rightarrow \mathrm{S}\left(\mathbf{N}, \mathrm{L}_{2}(0, T ; \mathcal{Z})\right), \quad \Omega \zeta=\hat{\zeta},
$$

where $\hat{\zeta}=\left(\hat{\zeta}_{k}: k \in \mathbf{N}\right)$, with $\mathrm{S}(\mathbf{N}, \mathcal{S})$ the space of $\mathcal{S}$-valued sequences and

$$
\hat{\zeta}_{k} \triangleq \zeta(k T+\cdot) \in \mathrm{L}_{2}(0, T ; \mathcal{Z})
$$




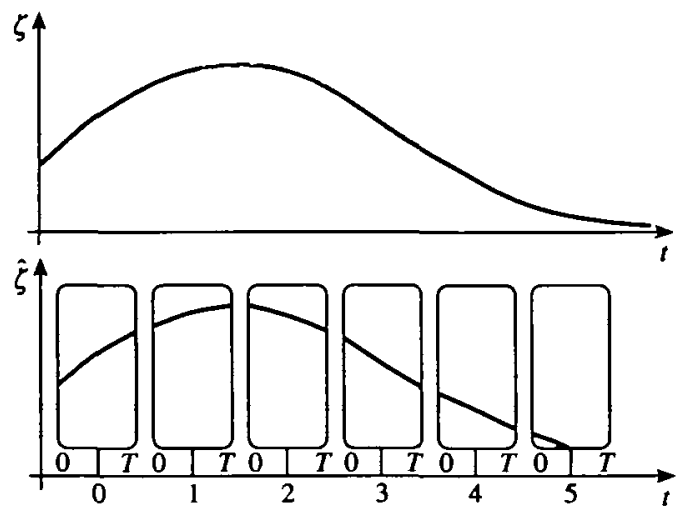

Fig. 11. Lifting a $\mathrm{L}_{2}\left(\mathbf{R}_{+}, \mathcal{Z}\right)$ signal to a $\ell_{2}\left(\mathbf{N}, \mathrm{L}_{2}(0, T ; \mathcal{Z})\right)$ sequence.

It is easy to show (see $[7,39]$ for technical details of the proof) that $\Omega$ is a linear bijective isometry from $\mathrm{L}_{2}^{\text {loc }}\left(\mathbb{R}_{+}, \mathcal{Z}\right)$ to $\mathrm{S}\left(\mathrm{N}, \mathrm{L}_{2}(0, T ; \mathcal{Z})\right)$. Notice also that, if $\zeta(\cdot)$ is a piecewise constant function satisfying $\zeta(t)=\zeta(k T)$ for $t \in[k T, k T+T)$, then $\hat{\zeta}$ may be represented by the sample sequence $\hat{\zeta}=(\zeta(k T): k \in \mathbf{N})$.

We shall apply the lifting technique to the smooth Pritchard-Salamon system

$$
\Sigma\left(S(\cdot),\left[B_{1}, B_{2}\right],\left[\begin{array}{l}
C_{1} \\
C_{2}
\end{array}\right],\left[\begin{array}{cc}
D_{11} & D_{12} \\
0 & 0
\end{array}\right]\right)
$$

with respect to $\mathcal{W} \hookrightarrow \mathcal{V}$. Assume that $S(\cdot)$ is exponentially stable on $\mathcal{W}$, and consider

$$
x_{0} \in \mathcal{W} \text {. }
$$

This fact implies that the measured output function is continuous with respect to time. We assume that $u_{2}$ is constant on $[k T, k T+T)$ for all $k \in \mathbf{N}$. Then the lifted $u_{2}(\cdot)$ is representable by the sample sequence $\tilde{u}_{2}$, and hence we may take $\hat{u}_{2}=\tilde{u}_{2} \in \ell_{2}\left(\mathbb{N}, \mathcal{U}_{2}\right)$.

Let us apply the lifting technique defined above in this section. The state equation written at the sampling instants becomes

$$
x(k T+T)=\Phi x(k T)+\hat{\Gamma}_{1} \hat{u}_{1, k}+\Gamma_{2} u_{2}(k T)
$$

with

$$
\begin{aligned}
& \Phi: \mathcal{W} \rightarrow \mathcal{W}, \quad \Phi \triangleq S^{\mathcal{W}}(T), \\
& \hat{\Gamma}_{1}: \mathrm{L}_{2}\left(0, T ; \mathcal{U}_{1}\right) \rightarrow \mathcal{W}, \quad \hat{\Gamma}_{1} \hat{u}_{1, k} \triangleq \int_{0}^{T} S^{\mathcal{V}}(T-\tau) B_{1} \hat{u}_{1, k}(\tau) \mathrm{d} \tau \\
& \Gamma_{2}: \mathcal{U}_{2} \rightarrow \mathcal{W}, \quad \Gamma_{2} u_{2} \triangleq \int_{0}^{T} S^{\mathcal{V}}(T-\tau) B_{2}(\tau) \mathrm{d} \tau u_{2} .
\end{aligned}
$$

The equation for the controlled output is now

$$
\hat{y}_{1}(k)=\hat{\Lambda}_{1} x(k T)+\hat{\theta}_{11} \hat{u}_{1, k}+\hat{\theta}_{12} u_{2}(k T)
$$


with the operators

$$
\begin{gathered}
\hat{\Lambda}_{1}: \mathcal{W} \rightarrow \mathrm{L}_{2}\left(0, T ; \mathcal{Y}_{1}\right), \\
\hat{\Theta}_{11}: \mathrm{L}_{2}\left(0, T ; \mathcal{U}_{1}\right) \rightarrow \mathrm{L}_{2}\left(0, T ; \mathcal{Y}_{1}\right), \quad \hat{\Theta}_{12}: \mathcal{U}_{2} \rightarrow \mathrm{L}_{2}\left(0, T ; \mathcal{Y}_{1}\right),
\end{gathered}
$$

defined by

$$
\begin{aligned}
\hat{\Lambda}_{1} & \triangleq C_{1} S^{\mathcal{W}}(\cdot), \\
\hat{\theta}_{11} \hat{u}_{1, k} & \triangleq C_{1} \int_{0}^{\cdot} S^{\mathcal{V}}(\cdot-\tau) B_{1} \hat{u}_{1, k}(\tau) \mathrm{d} \tau \\
\hat{\theta}_{12} u_{2}(k T) & \triangleq C_{1} \int_{0}^{\cdot} S^{\mathcal{v}}(\cdot-\tau) B_{2} u_{2}(k T) \mathrm{d} \tau+D_{12} \hat{u}_{2}(k T) .
\end{aligned}
$$

With this we have obtained the following discrete-time representation for the original system

$$
\begin{aligned}
x(k T+T) & =\Phi x(k T)+\hat{\Gamma}_{1} \hat{u}_{1, k}+\Gamma_{2} u_{2}(k T), \\
\hat{y}_{1}(k) & =\hat{\Lambda}_{1} x(k T)+\hat{\theta}_{11} \hat{u}_{1, k}+\hat{\theta}_{12} u_{2}(k T), \\
y_{2}(k) & =\Lambda_{2} x(k T),
\end{aligned}
$$

where

$$
\begin{gathered}
\hat{u}_{1 .} \in \ell_{2}\left(\mathbf{N}, \mathrm{L}_{2}\left(0, T ; \mathcal{U}_{1}\right)\right), \quad u_{2}(\cdot T) \in \ell_{2}\left(\mathbf{N}, \mathcal{U}_{2}\right), \\
x(\cdot T) \in \ell_{2}(\mathbf{N}, \mathcal{W}), \\
\hat{y}_{1} \in \ell_{2}\left(\mathbf{N}, \mathbf{L}_{2}\left(0, T ; \mathcal{Y}_{1}\right)\right), \quad y_{2} \in \ell_{2}\left(\mathbf{N}, \mathcal{Y}_{2}\right) .
\end{gathered}
$$

Corollary 4.17 The operators in (4.10) satisfy the boundedness conditions

$$
\begin{aligned}
\Phi \in \mathfrak{I}(\mathcal{W}), & \\
\hat{\Gamma}_{1} \in \mathfrak{L}\left(\mathrm{L}_{2}\left(0, T ; \mathcal{U}_{1}\right), \mathcal{W}\right), & \Gamma_{2} \in \mathfrak{I}\left(\mathcal{U}_{2}, \mathcal{W}\right), \\
\hat{\Lambda}_{1} \in \mathfrak{L}\left(\mathcal{W}, \mathrm{L}_{2}\left(0, T ; \mathcal{Y}_{1}\right)\right), & \Lambda_{2} \in \mathfrak{I}\left(\mathcal{W}, \mathcal{Y}_{2}\right), \\
\hat{\theta}_{11} \in \mathfrak{L}\left(\mathrm{L}_{2}\left(0, T ; \mathcal{U}_{1}\right), \mathrm{L}_{2}\left(0, T ; \mathcal{Y}_{1}\right)\right), & \hat{\theta}_{12} \in \mathfrak{L}\left(\mathcal{U}_{2}, \mathrm{~L}_{2}\left(0, T ; \mathcal{Y}_{1}\right)\right)
\end{aligned}
$$

Proof. Notice first that the proof of the second item of Proposition $4.18 \mathrm{can}$, with minor modifications, be adjusted so that $\left.\hat{\Gamma}_{1} \in \mathfrak{L}\left(\mathrm{L}_{2}\left(0, T ; \mathcal{U}_{1}\right), \mathcal{W}\right)\right)$. Applying the result proved in the above-mentioned item to the pair $\left(S(\cdot), B_{2}\right)$, we also have the boundedness property $\Gamma_{z} \in \mathfrak{L}\left(\mathcal{U}_{2}, \mathcal{W}\right)$, which yields the result immediately by the definitions of $\hat{\Lambda}_{1}, \tilde{\theta}_{11}$, and $\hat{\theta}_{12}$.

4.2.2 The relationship with power stability. Let us give first the main result on hybrid stabilizability of Pritchard-Salamon systems by generalizing Theorem 1 from [11] for this class of systems. 
Proposition 4.18 Consider the smooth Pritchard-Salamon system (4.8) and assume that the following hold: (i) $\left(S(\cdot), B_{2}\right)$ is admissibly stabilizable, (ii) $\left(C_{2}, S(\cdot)\right)$ is admissibly detectable, (iii) $\mathcal{U}_{2}=\mathbb{R}^{m_{2}}$, and (iv) $\mathcal{Y}_{2}=\mathbb{R}^{P_{2}}$. Then a digital controller $\Sigma_{\mathrm{K}}\left(S_{\mathrm{c}}(\cdot), B_{\mathrm{c}}, C_{\mathrm{c}}, D_{\mathrm{c}}\right)$ of sampling period $T$ provides hybrid stability for $(4.8)$ if it provides power stability for the discrete-time system that maps $\tilde{u}_{2}$ to $\tilde{y}_{2}$.

Proof. For the proof, the reader is referred to [2].

The digital control system, hybrid in nature, becomes after lifting a discrete-time one. The resulting closed-loop system is then

$$
x_{\mathrm{R}}(k+1)=\Phi_{\mathrm{R}} \cdot x_{\mathrm{R}}(k)+\Gamma_{\mathrm{R}} u_{\mathrm{R}}(k), \quad y_{\mathrm{R}}(k)=\Lambda_{\mathrm{R}} x_{\mathrm{R}}(k)+\theta_{\mathrm{R}} u_{\mathrm{R}}(k),
$$

where

$$
\begin{gathered}
x_{\mathrm{R}}(k)=\left[\begin{array}{c}
x(k T) \\
\xi(k)
\end{array}\right] \quad(k \in \mathbb{N}) \\
\Phi_{\mathrm{R}}=\left[\begin{array}{cc}
\Phi+\Gamma_{2} D_{\mathrm{c}} C_{2} & \Gamma_{2} C_{\mathrm{c}} \\
B_{\mathrm{c}} \Lambda_{2} & A_{\mathrm{c}}
\end{array}\right], \quad \Gamma_{\mathrm{R}}=\left[\begin{array}{c}
\hat{\Gamma} \\
0
\end{array}\right], \\
\Lambda_{\mathrm{R}}=\left[\hat{\Lambda}_{1}+\hat{\theta}_{12} D_{\mathrm{c}} \Lambda_{2}, \hat{\theta}_{12} C_{\mathrm{c}}\right], \quad \theta_{\mathrm{R}}=\hat{\theta}_{11}
\end{gathered}
$$

It is rather straightforward that, if $\mathcal{X}_{\mathrm{R}}=\mathcal{W} \oplus \mathcal{X}_{\mathrm{c}}$ stands for the augmented state space for the resultant closed-loop system, then the following boundedness conditions are satisfied:

$$
\begin{aligned}
& \Phi_{\mathrm{R}} \in \mathfrak{I}\left(\mathcal{X}_{\mathrm{R}}\right), \quad \Gamma_{\mathrm{R}} \in \mathfrak{I}\left(\mathrm{L}_{2}\left(0, T ; \mathcal{U}_{1}\right), \mathcal{X}_{\mathrm{R}}\right), \\
& \Lambda_{\mathrm{R}} \in \mathfrak{I}\left(\mathcal{X}_{\mathrm{R}}, \mathrm{L}_{2}\left(0, T ; \mathcal{Y}_{1}\right)\right), \quad \theta_{\mathrm{R}} \in \mathfrak{I}\left(\mathrm{L}_{2}\left(0, T ; \mathcal{U}_{1}\right), \mathrm{L}_{2}\left(0, T ; \mathcal{Y}_{1}\right)\right)
\end{aligned}
$$

The following proposition establishes the equivalence between the hybrid stability of a Pritchard-Salamon system and the power stability of its lifted counterpart [3].

Proposition 4.19 Let us consider smooth Pritchard-Salamon system (4.8) such that the hypotheses (i)-(iv) of Proposition 4.18 hold. Then the system (4.8) it is hybrid-stabilizable if and only if the lifted closed-loop system dynamics operator

$$
\Phi_{\mathrm{R}}=\left[\begin{array}{cc}
\Phi+\Gamma_{2} N \Lambda_{2} & \Gamma_{2} M \\
L \Lambda_{2} & K
\end{array}\right]
$$

is power-stable on $\mathcal{W}_{\mathrm{R}}=\mathcal{W} \oplus \mathcal{X}_{\mathrm{c}}$.

Having results available on lifting a Pritchard-Salamon system, we conclude this section by giving a result which shows that the lifting is indeed an appropriate tool for solving the digital $\mathrm{H}^{x}$ control problem. Clearly, the following holds.

Proposition 4.20 Let

$$
G_{1}: \mathbf{L}_{2}^{\text {loc }}\left(\mathbb{R}_{+}, \mathcal{U}_{1}\right) \rightarrow \mathbf{L}_{2}^{\text {loc }}\left(\mathbb{R}_{+}, \mathcal{Y}_{1}\right)
$$


be the input-output operator from $u_{1}$ to $y_{1}$, and let

$$
\hat{G}_{1}: \ell_{2}\left(\mathrm{~N}, \mathrm{~L}_{2}\left(0, T: \mathcal{U}_{1}\right)\right) \rightarrow \ell_{2}\left(\mathbf{N}, \mathrm{L}_{2}\left(0, T ; \mathcal{Y}_{1}\right)\right)
$$

be the input-output operator from $\hat{u}_{1}$ to $\hat{v}_{1}$. Then

$$
\left\|G_{1}\right\|=\left\|\hat{G}_{1}\right\| \text {. }
$$

\section{The equivalent discrete-time control problem}

Taking into account the prestabilization results proved in the previous section, we can assume, without loss of generality, that the Pritchard-Salamon-Popov triple $\Sigma\left(S^{\mathcal{V}}(\cdot), B, M\right)$ has its $\mathrm{C}_{0}$ semigroup exponentially stable on $\mathcal{W}$. In this section we show how the digital control problem can be converted into an equivalent discrete-time control problem.

\subsection{The equivalent discrete-time $L Q$ control problem}

Lemma 5.1 Let

$$
\Sigma\left(S^{\mathcal{\nu}}(\cdot), B, M=\left[\begin{array}{cc}
Q & L \\
L^{*} & R
\end{array}\right]=M^{*}\right)
$$

be a smooth Pritchard-Salamon-Popov triple (see Definitions 2.7 and 2.9) with respect to $(\mathcal{W} \hookrightarrow \mathcal{V}, \mathcal{U})$. Assume that $\mathcal{U}=\mathbb{R}^{m}$ and let $x_{0} \in \mathcal{W}$. Let $\left(x_{0}, x_{1}, \ldots, x_{k}=\right.$ $x(k T), \ldots)$ be the sampled state sequence and let $u_{\text {step }} \in L_{2}\left(\mathbb{R}_{+}, \mathcal{U}\right)$ be a piecewise constant control function: $u_{\text {step }}(t)=\mathrm{H}_{T} u(k)(k T \leqslant t<k T+T)$. Then the triple and its associated initial-value problem, given by

$$
\dot{x}(t)=A^{\mathcal{W}} x(t)+B u_{\text {step }}(t), \quad x(0)=x_{0} \in \mathcal{W}
$$

admits, at sampling instants $k T(k \in \mathbf{N})$ a discrete-time representation via the equivalent discrete-time Popov triple

$$
\Sigma\left(\Phi, \Gamma, M_{\mathrm{T}}=\left[\begin{array}{ll}
Q_{\mathrm{T}} & L_{\mathrm{T}} \\
L_{\mathrm{T}}^{*} & R_{\mathrm{T}}
\end{array}\right]=M_{\mathrm{T}}^{*}\right)
$$

and the difference equation

$$
x_{k}=\Phi x_{k}+\Gamma u(k), \quad x_{0} \in \mathcal{W}
$$

where $(\Phi, \Gamma)$ are defined by $(4.6 \mathrm{a}, \mathrm{b})$ and

$$
M_{\mathrm{T}}=\left[\begin{array}{ll}
Q_{\mathrm{T}} & L_{\mathrm{T}} \\
L_{\mathrm{T}}^{*} & R_{\mathrm{T}}
\end{array}\right]=M_{\mathrm{T}}^{*} \in \mathfrak{I}(\mathcal{W} \oplus \mathcal{U}),
$$

with $Q_{\mathrm{T}} \in \mathfrak{Q}(\mathcal{W}), L_{\mathrm{T}}^{*} \in \mathfrak{Q}(\mathcal{W}, \mathcal{U})$, and $R_{\mathrm{T}} \in \mathfrak{L}(\mathcal{U})$ defined by

$$
\left\langle Q_{\mathrm{T}} x, y\right\rangle_{\mathcal{W}} \triangleq \int_{0}^{T}\left\langle Q S^{\mathcal{W}}(t) x, S^{\mathcal{W}}(t) y\right\rangle_{\mathcal{W}} \mathrm{d} t
$$




$$
\begin{aligned}
\left\langle L_{\mathrm{T}}^{*} x, u\right\rangle_{u} \triangleq & \int_{0}^{T}\left\langle L^{*} S^{\mathcal{W}}(t) x, u\right\rangle_{u} \mathrm{~d} t \\
& +\int_{0}^{T}\left\langle Q S^{\mathcal{W}}(t) x, \int_{0}^{t} S^{\mathcal{V}}(t-\tau) B u \mathrm{~d} \tau\right\rangle_{\mathcal{W}} \mathrm{d} t \\
\left\langle R_{\mathrm{T}} u, v\right\rangle_{u} \triangleq & T\langle R u, v\rangle_{u}+2 \int_{0}^{T}\left\langle L^{*} \int_{0}^{t} S^{\mathcal{V}}(t-\tau) B u \mathrm{~d} \tau, v\right\rangle_{u} \mathrm{~d} t \\
& +\int_{0}^{T}\left\langle Q \int_{0}^{t} S^{\mathcal{V}}(t-\tau) B u \mathrm{~d} \tau, \int_{0}^{t} S^{\mathcal{V}}(t-\tau) B v \mathrm{~d} \tau\right\rangle_{\mathcal{W}} \mathrm{d} t
\end{aligned}
$$

Proof. For the proof, the reader is referred to [2].

Since $S^{\mathcal{V}}(\cdot)$ was assumed to be exponentially stable on $\mathcal{W}$, it follows by Theorem 4.10 that $\Phi$ is power-stable on $\mathcal{W}$. Hence we can state now the main digital control result of this section.

THEOREM 5.2 Let

$$
\Sigma\left(S^{\mathcal{\nu}}(\cdot), B, M=\left[\begin{array}{ll}
Q & L \\
L^{*} & R
\end{array}\right]=M^{*}\right)
$$

be a Pritchard-Salamon-Popov triple on $(\mathcal{W} \hookrightarrow \mathcal{V}, \mathcal{U})$. Assume that $\mathcal{U}=\mathbb{R}^{m}$ and let $x_{0} \in \mathcal{W}$. Consider

$$
\begin{aligned}
& \tilde{R}_{\mathrm{T}}: \ell_{2}(\mathbf{N}, \mathcal{U}) \rightarrow \ell_{2}(\mathbf{N}, \mathcal{U}), \quad \tilde{R}_{\mathrm{T}} \triangleq \underline{R}_{\mathrm{T}}+\underline{L}_{\mathrm{T}}^{*} \Psi+\Psi^{*} \underline{L}_{\mathrm{T}}+\Psi^{*} \underline{Q}_{\mathrm{T}} \Psi, \\
& \Psi: \ell_{2}(\mathbf{N}, \mathcal{U}) \rightarrow \mathcal{W}, \quad(\Psi u)(k) \triangleq \sum_{i=0}^{k-1} \Phi^{k-i-1} \Gamma u(i),
\end{aligned}
$$

where the underline denotes the canonical extension of the operator to sequences, e.g. $\underline{L}_{\mathrm{T}}\left(u_{k}: k \in \mathbb{N}\right) \triangleq\left(L_{\mathrm{T}} u_{k}: k \in \mathbb{N}\right)$, with $\Phi, \Gamma$, and

$$
M_{\mathrm{T}}=\left[\begin{array}{ll}
Q_{\mathrm{T}} & L_{\mathrm{T}} \\
L_{\mathrm{T}}^{*} & R_{\mathrm{T}}
\end{array}\right]
$$

defined by (4.6a), (4.6b), and $(5.1 \mathrm{a}, \mathrm{b}, \mathrm{c})$ respectively. If $\tilde{R}_{\mathrm{T}}^{-1}$ is well defined and bounded, then the digital optimal-control problem admits a solution in the sense that

$$
\begin{aligned}
\min _{u_{\text {step }}(\cdot) \in \mathcal{U}_{\text {com }}^{x_{0}}} J_{\Sigma}\left(x_{0}, u(\cdot)\right) & =\min _{u_{\text {step }}(\cdot) \in u_{\text {dm }}^{x_{0}}} \sum_{k=0}^{\infty}\left\langle\left[\begin{array}{l}
x(k) \\
u(k)
\end{array}\right],\left[\begin{array}{ll}
Q_{\mathrm{T}} & L_{\mathrm{T}} \\
L_{\mathrm{T}}^{*} & R_{\mathrm{T}}
\end{array}\right]\left[\begin{array}{l}
x(k) \\
u(k)
\end{array}\right]\right\rangle_{\mathcal{W} \times u} \\
& =\left\langle X x_{0}, x_{0}\right\rangle_{\mathcal{W}},
\end{aligned}
$$

where $u_{\text {step }}(t)=u(k)(k T \leqslant t<k T+T)$, and where $X \in \mathfrak{L}(\mathcal{W})$ is a stabilizing solution to the discrete-time Riccati equation

$$
\Phi^{*} X \Phi-X-\left(\Phi^{*} X \Gamma+L_{\mathrm{T}}\right)\left(R_{\mathrm{T}}+\Gamma^{*} X \Gamma\right)^{-1}\left(L_{\mathrm{T}}^{*}+\Gamma^{*} X \Phi\right)+Q_{\mathrm{T}}=0 .
$$

The minimum is attained for inputs of the form

$$
u_{\text {step }}(t)=\left(R_{\mathrm{T}}+\Gamma^{*} X \Gamma\right)^{-1}\left(L_{\mathrm{T}}^{*}+\Gamma^{*} X \Phi\right) x(k) \quad(k T \leqslant t<k T+T) .
$$




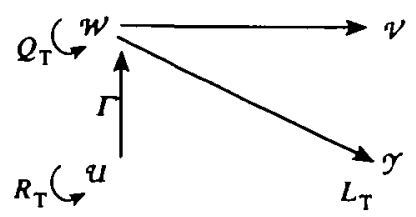

Fig. 12. The mappings generated by the discrete Popov triple $\Sigma\left(\Phi, \Gamma, M_{\mathrm{T}}\right)$.

REMARK 5.3 Notice that discretization has a 'smoothing' effect on the discrete Popov triple $\Sigma\left(\Phi, \Gamma, M_{\mathrm{T}}\right)$ as shown by the mapping representation in Fig. 12.

\subsection{The case $x_{0} \in \mathcal{V}$}

From the very beginning of this paper, we have considered that the initial state $x_{0}$ belongs to the smaller space $\mathcal{W}$, and we believe that we have succeeded in offering the reader a reasonable motivation for such an option. From the mathematical point of view, a counterpart of Lemma 5.1 corresponding to the weaker assumption $x_{0} \in \mathcal{V}$ would probably be more beautiful and interesting. This is especially true since working on the bigger space $\mathcal{V}$ obliges one to overcome certain difficulties and to make use of specific techniques. For a comprehensive treatment of the LQ optimal control problem in the case $x_{0} \in \mathcal{V}$, the reader is referred to $[14,34,37]$. In this section we use the technique developed in $[14,37]$ to extend the result proved in Lemma 5.1 to the case when the initial state belongs to the bigger state space $\mathcal{V}$.

Let us consider the Pritchard-Salamon-Popov triple (2.11). Associated with it are the initial-value problem (2.12), the class of admissible control functions (2.13), and the quadratic cost function (2.14). Recall that, if $x_{0} \in \mathcal{V}$, then the mild solution to the initial-value problem is a $\mathcal{V}$-valued function which is continuous with respect to the time $t$. Furthermore, as we have noticed earlier in Remark 4.8, the time-discretized state function is well defined as a $\mathcal{V}$-valued sequence and, at the sampling instants $k T(k \in \mathbb{N})$, the initial-value problem (2.12) admits a discrete-time representation of the form

$$
x(k T+T)=\Phi x(k T)+\Gamma u(k T), \quad x(0)=x_{0} \in \mathcal{V}
$$

but where now $\Phi$ and $\Gamma$ are defined by (4.7a) and (4.7b) respectively. Our goal is to derive an equivalent discrete-time Pritchard-Salamon-Popov triple when $x_{0} \in \mathcal{V}$. That means we want to construct an operator

$$
\overline{M_{\mathrm{T}}}=\left[\begin{array}{cc}
\overline{Q_{\mathrm{T}}} & \left(\overline{L_{\mathrm{T}}^{*}}\right) \\
\overline{L_{\mathrm{T}}^{*}} & R_{\mathrm{T}}
\end{array}\right]=M_{\mathrm{T}}^{*} \in \mathfrak{L}(\mathcal{V} \oplus \mathcal{U}),
$$

with $\overline{Q_{\mathrm{T}}} \in \mathfrak{L}(\mathcal{V}), \overline{L_{\mathrm{T}}^{*}} \in \mathfrak{L}(\mathcal{V}, \mathcal{U})$, and $R_{\mathrm{T}} \in \mathfrak{L}(\mathcal{U})$ such that

$$
J_{\Sigma}\left(x_{0}, u(\cdot)\right)=\sum_{k=0}^{\infty}\left\langle\left[\begin{array}{l}
x(k T) \\
u(k T)
\end{array}\right],\left[\begin{array}{cc}
\overline{Q_{\mathrm{T}}} & \left(\overline{L_{\mathrm{T}}^{*}}\right)^{*} \\
\overline{L_{\mathrm{T}}^{*}} & R_{\mathrm{T}}
\end{array}\right]\left[\begin{array}{l}
x(k T) \\
u(k T)
\end{array}\right]\right\rangle_{\mathcal{V} \times u},
$$

where $J_{\Sigma}\left(x_{0}, u(\cdot)\right)$ is the corresponding quadratic index.

Since $Q \in \mathfrak{L}(\mathcal{W})$ is an admissible weighting operator, it follows that, for any 
$x, y \in \mathcal{W}$

$$
\left|\left\langle Q_{\mathrm{T}} x, y\right\rangle_{\mathcal{W}}\right|=\left|\int_{0}^{T}\left\langle Q S^{\mathcal{W}}(t) x, S^{\mathcal{W}}(t) y\right\rangle_{\mathcal{W}} \mathrm{d} t\right| \leqslant\|x\|_{\mathcal{V}}\|y\|_{\mathcal{V}}
$$

Since $\mathcal{W} \hookrightarrow \mathcal{V}$, it follows by applying Theorem 1 from p. 12 in [1] that the sesquilinear form (5.3) can be extended to a bounded sesquilinear form on $\mathcal{V}$. Thus let $\overline{Q_{\mathrm{T}}}=\left(\overline{Q_{\mathrm{T}}}\right)^{*} \in \mathfrak{L}(\mathcal{V})$ denote the self-adjoint operator associated with the extended sesquilinear form, so that

$$
\left\langle Q_{\mathrm{T}} x, y\right\rangle_{\mathcal{W}}=\left\langle\overline{Q_{\mathrm{T}}} x, y\right\rangle_{\mathcal{V}} \quad(x, y \in \mathcal{W})
$$

In a similar way we construct the extension of $L_{\mathrm{T}}^{*} \in \mathfrak{Q}(\mathcal{W}, \mathcal{U})$ to $\mathcal{V}$, denoted $\overline{L_{\tau}^{*}} \in \mathfrak{L}(\mathcal{V}, \mathcal{U})$. Notice first that the following hold.

(i) Since $L_{\mathrm{T}}^{*} \in \mathfrak{Q}(\mathcal{W}, \mathcal{U})$ is an admissible output operator for $S(\cdot)$ with respect to $W \hookrightarrow \mathcal{V}$, then there exists $\alpha>0$ such that

$$
\left|\int_{0}^{T}\left\langle L^{*} S^{\mathcal{W}}(t) x, u\right\rangle_{\mathcal{U}} \mathrm{d} t\right| \leqslant \alpha\|x\|_{\mathcal{V}}\|u\|_{\mathcal{U}}
$$

This is true since the left-hand side of (5.4) defines a continuous bilinear mapping from $\mathcal{W} \oplus \mathcal{U}$ to $\mathbb{R}$.

(ii) Since $B \in \mathfrak{L}(\mathcal{U}, \mathcal{V})$ is an admissible input operator for $S(\cdot)$ with respect to $\mathcal{W} \hookrightarrow \mathcal{V}$, then there exists $\beta>0$ such that

$$
\left\|\int_{0}^{t} S^{\mathcal{\nu}}(t-\tau) B u \mathrm{~d} \tau\right\|_{\mathcal{W}} \leqslant \beta\|u\|_{\mathcal{U}}
$$

for $t \in[0, T)$. It follows that

$$
\left|\int_{0}^{T}\left\langle Q S^{\mathcal{W}}(t) x, \int_{0}^{t} S^{\mathcal{V}}(t-\tau) B u \mathrm{~d} \tau\right\rangle_{\mathcal{W}}\right| \leqslant \beta\|x\|_{\mathcal{V}}\|u\|_{\mathcal{U}} .
$$

From (5.4) and (5.5) it follows that

$$
\begin{aligned}
\left|\left\langle L_{\mathrm{T}}^{*} x, u\right\rangle_{\mathcal{U}}\right| & =\left|\int_{0}^{T}\left\langle L^{*} S^{\mathcal{W}}(t) x, u\right\rangle_{\mathcal{U}} \mathrm{d} t+\left\langle Q S^{\mathcal{W}}(t) x, \int_{0}^{t} S^{\mathcal{V}}(t-\tau) B u \mathrm{~d} \tau\right\rangle_{\mathcal{W}}\right| \\
& \leqslant\left|\int_{0}^{T}\left\langle L^{*} S^{\mathcal{W}}(t) x, u\right\rangle_{\mathcal{U}} \mathrm{d} t\right|+\left|\int_{0}^{T}\left\langle Q S^{\mathcal{W}}(t) x, \int_{0}^{t} S^{\mathcal{V}}(t-\tau) B u \mathrm{~d} \tau\right\rangle_{\mathcal{W}} \mathrm{d} t\right| \\
& \leqslant \gamma\|x\|_{\mathcal{V}}\|u\|_{\mathcal{U}},
\end{aligned}
$$

for some appropriately chosen $\gamma>0$. The last inequality implies that the bilinear form $\left\langle L_{\mathrm{T}}^{*} x, u\right\rangle_{\mathcal{U}}$ on $\mathcal{W} \times \mathcal{U}$ has a continuous extension to a bilinear form on $\mathcal{V} \times \mathcal{U}$. Let $\overline{L_{\mathrm{T}}^{*}} \in \mathfrak{L}(\mathcal{V}, \mathcal{U})$ denote the operator associated with this extended bilinear form, so that

$$
\left\langle L_{\mathrm{T}}^{*} x, u\right\rangle_{\mathcal{U}}=\left\langle\overline{L_{\mathrm{T}}^{*}} x, u\right\rangle_{\mathcal{u}} \quad(x \in \mathcal{W}, u \in \mathcal{U})
$$

It remains to prove that $R_{\mathrm{T}}$ is well defined and bounded from $\mathcal{U}$ to $\mathcal{U}$. Since $L^{*}$ is an admissible output operator and $B$ is an admissible input operator, 
it follows that

$$
\begin{aligned}
\left|\left\langle R_{\mathrm{T}} u, u\right\rangle_{u}\right| \leqslant & \left|T\langle R u, u\rangle_{u}\right|+2\left|\int_{0}^{T}\left\langle L^{*} \int_{0}^{t} S^{\mathcal{V}}(t-\tau) B u \mathrm{~d} \tau, u\right\rangle_{u} \mathrm{~d} t\right| \\
& +\left|\int_{0}^{T}\left\langle Q \int_{0}^{t} S^{\mathcal{\nu}}(t-\tau) B u \mathrm{~d} \tau, \int_{0}^{t} S^{\mathcal{V}}(t-\tau) B u \mathrm{~d} \tau\right\rangle_{\mathcal{V}} \mathrm{d} t\right| \\
\leqslant & \mu\|u\|_{\mathcal{U}}^{2},
\end{aligned}
$$

for some appropriately chosen $\mu>0$.

An immediate fact which is implied by the construction of $\overline{Q_{\mathrm{T}}}=\left(\overline{Q_{\mathrm{T}}}\right)^{*} \in \mathfrak{L}(\mathcal{V})$ and $\overline{L_{\mathrm{T}}^{*}} \in \mathfrak{L}(\mathcal{V}, \mathcal{U})$ is the following.

Proposition 5.4 The quadratic functional $J_{\Sigma}\left(x_{0}, u(\cdot)\right)$ associated with the equivalent discrete-time Pritchard-Salamon-Popov triple

$$
\Sigma\left(\Phi, \Gamma, M_{\mathrm{T}}=\left[\begin{array}{ll}
Q_{\mathrm{T}} & L_{\mathrm{T}} \\
L_{\mathrm{T}}^{*} & R_{\mathrm{T}}
\end{array}\right]=M_{\mathrm{T}}^{*}\right),
$$

with the Popov index $M_{\mathrm{T}}$ defined by (5.1), has a unique bounded extension to a quadratic functional for $x_{0} \in \mathcal{V}$ which can be effectively constructed. Furthermore,

$$
\begin{aligned}
J_{\Sigma}\left(x_{0}, u(\cdot)\right) & =\sum_{k=0}^{\infty}\left\langle\left[\begin{array}{l}
x(k T) \\
u(k T)
\end{array}\right],\left[\begin{array}{ll}
Q_{\mathrm{T}} & L_{\mathrm{T}} \\
L_{\mathrm{T}}^{*} & R_{\mathrm{T}}
\end{array}\right]\left[\begin{array}{l}
x(k T) \\
u(k T)
\end{array}\right]\right\rangle_{\mathcal{W} \times \mathcal{U}} \\
& =\sum_{k=0}^{\infty}\left\langle\left[\begin{array}{l}
x(k T) \\
u(k T)
\end{array}\right],\left[\begin{array}{cc}
\overline{Q_{\mathrm{T}}} & \left(\overline{L_{\mathrm{T}}^{*}}\right)^{*} \\
\overline{L_{\mathrm{T}}^{*}} & R_{\mathrm{T}}
\end{array}\right]\left[\begin{array}{l}
x(k T) \\
u(k T)
\end{array}\right]\right\rangle_{\mathcal{V} \times u} .
\end{aligned}
$$

Let us also notice that $\Sigma\left(\Phi, \Gamma, \overline{M_{\mathrm{T}}}\right)$, where

$$
\overline{M_{\mathrm{T}}}=\left[\begin{array}{cc}
\overline{Q_{\mathrm{T}}} & \left(\overline{L_{\mathrm{T}}^{*}}\right)^{*} \\
\overline{L_{\mathrm{T}}^{*}} & R_{\mathrm{T}}
\end{array}\right]=M_{\mathrm{T}}^{*} \in \mathfrak{L}(\mathcal{V} \oplus \mathcal{U}),
$$

with $\Phi$ and $\Gamma$ defined by (4.7) and $\overline{M_{\mathrm{T}}}$ constructed as before, is a well defined discretetime Popov triple on $(\mathcal{V}, \mathcal{U})$. Let us consider then the digital control problem on $\mathcal{V}$. By appropriately modifying the definition of the stabilizing digital control law (Definition 2.12), by considering as the set of admissible control functions the one defined by (2.13), and finally by considering the quadratic cost function defined by the extended quadratic functional, we can ensure that the digital control problem on $\mathcal{V}$ is well-posed. This is especially true since, assuming the stability of $S(\cdot)$ also on $\mathcal{V}$, we can write down without any difficulty the counterpart of the digital control result from Theorem 5.2 for the case when $x(t)$ evolves in $\mathcal{V}$. Notice that, this time, the solution $\bar{X} \in \mathfrak{L}(\mathcal{V})$ to the following digital Riccati equation is involved in the expression of the digital stabilizing control law:

$$
\Phi^{*} X \Phi-X-\left[\Phi^{*} X \Gamma+\left(\overline{L_{\mathrm{T}}^{*}}\right)^{*}\right]\left(R_{\mathrm{T}}+\Gamma^{*} X \Gamma\right)^{-1}\left(\overline{L_{\mathrm{T}}^{*}}+\Gamma^{*} X \Phi\right)+\overline{Q_{\mathrm{T}}}=0
$$

with $\Phi$ and $\Gamma$ defined by (4.7). Furthermore, the minimum of the quadratic cost 
functional is attained, and its value is

$$
\min _{u(\cdot) \in \mathcal{U}_{\operatorname{sim}}^{0} \cap \mathrm{PC}_{r}\left(\mathbb{R}_{+}, u\right)} J_{\Sigma}\left(x_{0}, u(\cdot)\right)=\left\langle\bar{X} x_{0}, x_{0}\right\rangle_{\mathcal{V}}
$$

When $x(t)$ evolves in $\mathcal{W}$, then the above minimum of the quadratic functional is attained at $\left\langle X x_{0}, x_{0}\right\rangle_{\mathcal{W}}$, but regarding $x(t) \in \mathcal{W}$ as element of $\mathcal{V}$ shows that it is attained at $\left\langle\bar{X} x_{0}, x_{0}\right\rangle_{\mathcal{V}}$. Hence, by exploiting the result of Proposition 5.4 we conclude that

$$
\left\langle X x_{0}, x_{0}\right\rangle_{\mathcal{W}}=\left\langle\bar{X} x_{0}, x_{0}\right\rangle_{\mathcal{V}} \quad\left(x_{0} \in \mathcal{W}\right) .
$$

This fact implies that $\bar{X}=\bar{X}^{*} \in \mathfrak{L}(\mathcal{V})$ is the unique bounded extension to $\mathcal{V}$ of $X=X^{*} \in \mathfrak{L}(\mathcal{W})$.

\subsection{The digital $\mathrm{H}^{\infty}$ control problem}

Consider the continuous dense injection of spaces $\mathcal{W} \hookrightarrow \mathcal{X} \hookrightarrow \mathcal{V}$, assume that there exist two other real separable Hilbert spaces $\mathcal{U}_{1}$ and $\mathcal{Y}_{1}$, and let $B_{1} \in \mathfrak{Q}\left(\mathcal{U}_{1}, \mathcal{V}\right)$, $B_{2} \in \mathfrak{L}\left(\mathcal{U}_{2}, \mathcal{V}\right), C_{1} \in \mathfrak{I}\left(\mathcal{W}, \mathcal{Y}_{1}\right), C_{2} \in \mathfrak{L}\left(\mathcal{W}, \mathcal{Y}_{2}\right)$, and $D_{12} \in \mathfrak{I}\left(\mathcal{U}_{2}, \mathcal{Y}_{1}\right)$. We consider the smooth Pritchard-Salamon system

$$
\Sigma\left(S(\cdot),\left[B_{1}, B_{2}\right],\left[\begin{array}{l}
C_{1} \\
C_{2}
\end{array}\right],\left[\begin{array}{cc}
D_{11} & D_{12} \\
0 & 0
\end{array}\right]\right)
$$

satisfying

$$
\begin{aligned}
x(t) & =S^{\mathcal{W}}(t) x_{0}+\int_{0}^{t} S^{\mathcal{V}}(t-\tau)\left[B_{1} u_{1}(\tau)+B_{2} u_{2}(\tau)\right] \mathrm{d} \tau \\
y_{1}(t) & =C_{1} x(t)+D_{11} u_{1}(t)+D_{12} u_{2}(t), \quad y_{2}(t)=C_{2} x(t) .
\end{aligned}
$$

In our set-up, $x(t) \in \mathcal{W}$ is the state, $u_{1}(t) \in \mathcal{U}_{1}$ is the disturbance input $u_{2}(t) \in \mathcal{U}_{2}$ is the control input, $y_{1}(t) \in \mathcal{Y}_{1}$ the controlled output, and $y_{2}(t) \in \mathcal{Y}_{2}$ is the measured output. The following assumptions are made on the Pritchard-Salamon system:

(A1) $\mathcal{U}_{2}=\mathbb{R}^{m}$ (we have $m$ actuators),

(A2) $\mathcal{Y}_{2}=\mathbb{R}^{p}$ (we have $p$ sensors),

(A3) $x_{0} \in \mathcal{W}$ (the output measurement is a continuous $\mathcal{Y}$-valued function),

(A4) $\left(S(\cdot), B_{2}\right)$ is admissibly stabilizable,

(A5) $\left(C_{2}, S(\cdot)\right)$ is admissibly detectable,

(A6) the operators

$$
\left[\begin{array}{cc}
\mathrm{z} I-A & -B_{2} \\
C_{1} & D_{12}
\end{array}\right] \text { and }\left[\begin{array}{cc}
\mathrm{z} I-A & -B_{1} \\
C_{2} & 0
\end{array}\right]
$$

are injective and surjective respectively.

Let $\mathcal{X}_{\mathrm{c}}$ be another real separable Hilbert space and consider a controller $\Sigma_{\mathrm{c}}\left(A_{\mathrm{c}}, B_{\mathrm{c}}, C_{\mathrm{c}}, D_{\mathrm{c}}\right)$ of the form

$$
(\mathrm{s} \xi)(k)=A_{\mathrm{c}} \xi(k)+B_{\mathrm{c}} \nu(k), \quad \zeta(k)=C_{\mathrm{c}} \xi(k)+D_{\mathrm{c}} \nu(k),
$$


where $\mathbf{s}$ is the forward shift operator $\mathrm{s}: \xi \mapsto \xi(\bullet+1), A_{\mathrm{c}} \in \mathfrak{L}\left(\mathcal{X}_{\mathrm{c}}\right), B_{\mathrm{c}} \in \mathfrak{L}\left(\mathbb{R}^{p}, \mathcal{X}_{\mathrm{c}}\right)$, $C_{\mathrm{c}} \in \mathcal{Q}\left(\mathcal{X}_{\mathrm{c}} \cdot \mathbb{R}^{m}\right), D_{\mathrm{c}} \in \mathbb{R}^{p \times m}$, and the initial state of the controller, $\xi_{0}$, is given. Since the output measurement is a $\mathbb{R}^{p}$-valued continuous function, we can define its time-discretized version as

$$
\tilde{y}_{2}=\mathrm{S}_{T} y_{2} \text {. }
$$

We want to make sense of the feedback connection

$$
\nu=\mathrm{S}_{2}^{\prime}, \quad u_{2}(\cdot)=\mathrm{H}_{T} \tilde{\zeta}
$$

Our goal is to design a controller (5.6) such that the following properties hold.

(i) (Stability) The closed-loop system $\Sigma_{\mathrm{GsdG}}$, obtained by the feedback interconnection of the hybrid generalized plant $G_{\mathrm{sd}}$ and the controller $G_{\mathbf{c}}$ is hybrid stable.

(ii) (Attenuation) The input-output operator from $u_{1}$ to $y_{1}$, i.e.

$$
G_{1}: \mathrm{L}_{2}\left(\mathbb{R}_{+}, \mathcal{U}_{1}\right) \mapsto \mathrm{L}_{2}\left(\mathbb{R}_{+}, \mathcal{Y}_{1}\right)
$$

expressed as a linear fractional transformation of the hybrid generalized plant $\Sigma_{\text {Gsd }}$, with input-output operator

$$
G_{\mathrm{sd}}=\left[\begin{array}{cc}
G_{11} & G_{12} \mathrm{H}_{T} \\
\mathrm{~S}_{T} G_{21} & \mathrm{~S}_{T} G_{22} \mathrm{H}_{T}
\end{array}\right]
$$

and the controller $\Sigma_{\mathrm{K}}$, with input-output operator $G_{\mathrm{c}}$

$$
G_{1}=\mathrm{F}\left(\left[\begin{array}{cc}
G_{11} & G_{12} \mathrm{H}_{T} \\
\mathrm{~S}_{T} G_{21} & \mathrm{~S}_{T} G_{22} \mathrm{H}_{T}
\end{array}\right], G_{\mathrm{c}}\right) \triangleq G_{11}+G_{12} \mathrm{H}_{T} G_{\mathrm{c}}\left(I-\mathrm{S}_{T} G_{22} \mathrm{H}_{T} G_{\mathrm{c}}\right)^{-1} \mathrm{~S}_{T} G_{21}
$$

is well defined and is a contraction:

$$
\left\|G_{1} u_{1}(\cdot)\right\|<1
$$

The following main conversion results hold (see [3]).

THEOREM 5.5 Let

$$
\Sigma\left(S(\cdot),\left[B_{1}, B_{2}\right],\left[\begin{array}{c}
C_{1} \\
C
\end{array}\right],\left[\begin{array}{cc}
0 & D_{12} \\
0 & 0
\end{array}\right]\right)
$$

be a smooth Pritchard-Salamon system with respect to $\mathcal{W} \hookrightarrow \mathcal{X} \hookrightarrow \mathcal{V}$ satisfying

$$
\Sigma_{\mathrm{G}}\left\{\begin{array}{l}
x(t)=S(t) x_{0}+\int_{0}^{t} S(t-\tau)\left[B_{1} u_{1}(\tau)+B_{2} u_{2}(\tau)\right] \mathrm{d} \tau, \quad x_{0} \in \mathcal{W}, \\
y_{1}(t)=C_{1} x(t)+D_{12} u_{2}(t), \quad y_{2}(t)=C_{2} x(t)
\end{array}\right.
$$

Then a discrete-time controller $\Sigma\left(A_{\mathrm{c}}, B_{\mathrm{c}}, C_{\mathrm{c}}, D_{\mathrm{c}}\right)$ satisfying

$$
\Sigma_{\mathrm{K}} \quad\left\{\begin{array}{l}
(\mathrm{s} \xi)(k)=A_{\mathrm{c}} \xi(k)+B_{\mathrm{c}} \eta(k) \\
\zeta(k)=C_{\mathrm{c}} \xi(k)+D_{\mathrm{c}} \eta(k)
\end{array}\right.
$$

solves the digital $\mathrm{H}^{x}$ control problem if it is a solution to the discrete-time $\mathrm{H}^{x}$ control problem for the lifted discrete-time system satisfying 


$$
\Sigma_{\hat{\mathrm{G}}}\left\{\begin{array}{lr}
x(k T+T)=\Phi x(k T)+\hat{\Gamma}_{1} \hat{u}_{1 . k}+\Gamma_{2} u_{2}(k T), \quad x(0)=x_{0} \in \mathcal{W} \\
\hat{y}_{1}(k)=\hat{\Lambda}_{1} x(k T)+\hat{\theta}_{11} \hat{u}_{1 . k}+\hat{\theta}_{12} u_{2}(k T), \quad y_{2 . k}=\Lambda_{2} x(k T),
\end{array}\right.
$$

where

$$
\begin{array}{cc}
\Phi \triangleq S^{\mathcal{V}}(T)\lceil\mathcal{W}, \\
\hat{\Gamma}_{1} \hat{u}_{1, k} \triangleq \int_{0}^{T} S^{\mathcal{\nu}}(T-\tau) B_{1} \hat{u}_{1, k}(\tau) \mathrm{d} \tau, \quad \Gamma_{2} \triangleq \int_{0}^{T} S^{\mathcal{V}}(T-\tau) B_{2} \mathrm{~d} \tau, \\
\hat{\Lambda}_{1} \triangleq C_{1} S^{\mathcal{V}}(\cdot), & \Lambda_{2} \triangleq C_{2}, \\
\hat{\theta}_{11} \hat{u}_{1, k} \triangleq C_{1} \int_{0}^{\cdot} S^{\mathcal{V}}(\cdot-\tau) B_{1} \hat{u}_{1, k}(\tau) \mathrm{d} \tau, & \hat{\theta}_{12} \triangleq C_{1} \int_{0}^{\bullet} S^{\mathcal{V}}(\cdot-\tau) B_{2} \mathrm{~d} \tau+D_{12},
\end{array}
$$

with $\hat{u}_{1, k}(\tau)=u(k T+\tau)(0 \leqslant \tau<T)$. Exploiting now the results on necessary and sufficient conditions for the existence of the solution to the discrete-time $\mathbf{H}^{\infty}$ control problem, stated and proved in Section 3, we can formulate the Popov-theory-based solution to the digital $\mathrm{H}^{\infty}$ control problem.

TheOREM 5.6 (i) If the digital $\mathrm{H}^{\infty}$ control problem admits a solution, then the KSPY systems $(3.15 \mathrm{a}, \mathrm{b})$ associated with the discrete-time Popov triples

$$
\begin{gathered}
\Sigma\left(\Phi,\left[\hat{\Gamma}_{1}, \Gamma_{2}\right],\left[\begin{array}{ccc}
\hat{\Lambda}_{1}^{*} \hat{\Gamma}_{1} & \hat{\Lambda}_{1}^{*} \hat{\theta}_{11} & \hat{\Lambda}_{1}^{*} \hat{\theta}_{12} \\
\hat{\theta}_{11}^{*} \hat{\Lambda}_{1} & \hat{\theta}_{11}^{*} \hat{\theta}_{11}-I & \hat{\theta}_{11}^{*} \hat{\theta}_{12} \\
\hat{\theta}_{12}^{*} \hat{\Lambda}_{1} & \hat{\theta}_{12}^{*} \hat{\theta}_{11} & \hat{\theta}_{12}^{*} \hat{\theta}_{12}
\end{array}\right]\right) \\
\Sigma\left(\Phi,\left[\hat{\Lambda}_{1}^{*}, \Lambda_{2}^{*}\right],\left[\begin{array}{ccc}
\hat{\Gamma}_{1} \hat{\Gamma}_{1}^{*} & \hat{\Gamma}_{1} \hat{\theta}_{11}^{*} & 0 \\
\hat{\theta}_{11} \hat{\Gamma}_{1}^{*} & \hat{\theta}_{11} \hat{\theta}_{11}^{*}-I & 0 \\
0 & 0 & 0
\end{array}\right]\right)
\end{gathered}
$$

have stabilizing solutions.

(ii) Consider the discrete-time Popov triple

$$
\Sigma_{\mathrm{O}}\left(\Phi_{\mathrm{O}},\left[\begin{array}{ll}
\hat{\Lambda}_{1 \mathrm{O}}^{*} & \Lambda_{2 \mathrm{O}}^{*}
\end{array}\right],\left[\begin{array}{ccc}
\hat{\Gamma}_{1 \mathrm{O}} \hat{\Gamma}_{1 \mathrm{O}}^{*} & \hat{\Gamma}_{1 \mathrm{O}} \hat{\Theta}_{11 \mathrm{O}}^{*} & 0 \\
\hat{\theta}_{110} \hat{\Gamma}_{1 \mathrm{O}}^{*} & \hat{\theta}_{110} \hat{\theta}_{11 \mathrm{O}}^{*}-I & 0 \\
0 & 0 & 0
\end{array}\right]\right)
$$

corresponding to the modified system defined by (3.20) associated with the lifted Pritchard-Salamon system (4.8). If the KSPY systems $(3.15 \mathrm{a}, \mathrm{b})$ written for the discrete-time Popov triples (5.7a) and (5.8) have stabilizing solutions, then there exists a controller which is a solution to the digital $\mathrm{H}^{x}$ control problem.

\section{Conclusions}

Discrete-time Popov theory gives necessary and sufficient conditions for the existence of a stabilizing solution to a discrete-time Riccati equation in terms of the invertibility of a Toeplitz operator associated with the discrete-time system. It represents the 
theoretical framework for this paper wherein we have approached the problem of digital control of infinite-dimensional systems with possible unbounded input and/or output operators. The framework of discrete Popov theory proves to be rich enough to permit one to write down the solutions to various discrete control problems, such as the LQ optimal-control problem and the $\mathrm{H}^{\infty}$ control problem with output measurement feedback respectively.

Having available the framework offered by this discrete-time control theory, we have approached the problem of digital control of linear infinite-dimensional systems with unbounded input and/or output operators of the Pritchard-Salamon type. Several concepts, specific to digital control, such as digital exponential stability and hybrid stability, have been analysed and discussed for systems with unboundedness and, consequently, they have been enriched with a new meaning, specific to such a framework, and have attained a fairly high degree of generality.

A modern concept, the so-called lifting technique, has represented the main tool that we used to write down an equivalent discrete-time control problem. A step of paramount importance in the process of applying the lifting technique to digital control was represented by Theorem 4.10 , where (among other results) it is shown that sampling and lifting have beneficial effects on the control structure in the sense that all the operators of the equivalent control structure are bounded on appropriate function spaces.

Another feature that has been captured in the picture offered by this survey is that the two discrete control theories applied here are suitable for coping in so-called singular control problems. These problems occur naturally in digital controlmostly due to the definition of the sample operator, which is undefined over $\mathrm{L}_{2}$ spaces of functions. An immediate example of a difficulty that we had to overcome is the singularity $D_{21}=0$ in the digital $\mathrm{H}^{\infty}$ control with measurement feedback. One of the most important contributions of this paper is given by the expression of some necessary and sufficient conditions for the existence of the suboptimal controller, achieving both hybrid stability and disturbance attenuation, in terms of the coercivity of input-output operators associated with the hybrid generalized control plant.

The last part of this paper was concerned with the applications. A general parabolic Pritchard-Salamon system was considered. We have computed the time-discretized counterparts as well as the equivalent discrete-time Pritchard-SalamonPopov triple. We have pointed out the difficulties arising in a possible implementation-difficulties that are mainly due to the need for calculating the inverse of a Toeplitz-like operator in order to express the stabilizing solution to a Riccati equation.

Let us try to point out the directions for future research opened by this study.

(i) First of all notice that the Pritchard-Salamon class of systems with unboundedness is not the most general one possible. It is sufficient to mention that Dirichlet boundary control usually leads to input operators that are 'too unbounded' for the Pritchard-Salamon framework. This obviously limits the generality of the digital control theory built up in $[4,3]$. In a series of papers $[30,31,35]$, Salamon and 
G. Weiss introduced the concepts of abstract linear systems and well posedness for linear systems and regular systems, respectively. Basically, regular systems are represented by

$$
\begin{aligned}
& \dot{x}(t)=A x(t)+B u(t), \quad x(0)=x_{0} \in \mathcal{X}, \\
& y(t)=C x(t)+D u(t),
\end{aligned}
$$

with $A, B$, and $C$ unbounded with respect to the Hilbert state space $\mathcal{X}$, and $D$ bounded from the space of inputs, say $\mathcal{U}$, to the space of outputs, $\mathcal{Y}$. An extension of the results of this paper such that they become applicable to the class of regular systems would be, in our opinion, not a trivial one. This statement is motivated as follows.

(a) In order to be sampled, the output function has to enjoy certain smoothness properties with respect to time. A sufficient condition (such as $x_{0} \in \mathcal{W}$ in the case of Pritchard-Salamon systems) for a smooth output function is not known yet for regular systems.

(b) Our main result on digital exponential stabilizability, Theorem 4.10, was proved on the basis of the structural decomposition. Such a decomposition might hold for regular systems with finite-dimensional input and output operators as well (we conjecture that this is indeed true), but for this class of systems the result is not available yet.

(c) A general Riccati theory for regular systems is not available yet either; the latest development of George Weiss [36] is about admissible feedback operators for regular systems. This is another reason why for this class of systems we cannot extend the main digital stabilizability result of this paper, Theorem 4.10.

(ii) Secondly, even if the results presented in this paper have apparently only a theoretical importance, they have (in our opinion) nevertheless a practical impact as well. Let us clarify this statement. A feature of paramount importance for implementation is the fact that the controller, solving a certain optimization control problem, is also an infinite-dimensional system. Such a controller, besides presenting the great disadvantage of endowing the system with unboundedness when obtained via a continuous-time synthesis, can be analytically determined only for particular examples. Even in this case, the physical process - the plant-has to be controlled, and the most convenient solution nowadays seems to be the employment of computer control. This implies that we have to represent the unbounded operators in a finite-stack computer memory-a typical discrete device. Such a representation would imply two operations made on the controller: approximation of the controller by a finite-dimensional one, and discretization. While, for finite-dimensional systems, those two operations can intertwine (as shown in [6]), this does not necessarily apply to infinite-dimensional systems generally. Besides the loss of optimality generated by approximating the controller, the fact that the two operations do not intertwine generates an additional uncertainty as to the degree of suboptimality of the real implementable controller. The digital-control approach we propose in this paper eliminates these disadvantages. The controller arises naturally from a digital control design process where the designer manipulates only bounded operators, and hence the outgrowth of such a design process is a discrete-time infinite-dimensional controller which is a system with bounded operators. The only residual major difficulty is the 
controller approximation. In this respect, the Popov-theory-based approach taken in this paper leads to approximation of a certain discrete Toeplitz operator. We believe that this opens a new and promising research direction: inverse Toeplitz operator theory applied to approximate stabilizing solutions to Riccati equations. There are basically two reasons why this direction is a promising one. The first is that the approximation of infinite-dimensional Riccati equations is usually done via ad-hoc methods (see Delfour [15] and the references therein), and hence there is no available theory-only some recipes. The second reason is an advantage of its intrinsic nature-obviating the difficulties caused by the ad-hoc nature of those methodsthat of having to approximate a Toeplitz operator, which is a task for which a very well developed literature is already available (see [23] and the references therein).

If we have succeeded in defining two major directions opened by this research - the extension of the main digital results to regular systems and the application of Toeplitz operator theory to obtaining approximations of solutions to digital Riccati equations-let us end this concluding section by highlighting some of the technical difficulties encountered that present, in our opinion, another source of interesting research. In subsection 5.3, we proved that a sufficient condition for the existence of the singular filtering $\mathrm{H}^{\infty}$ Riccati equation is represented by the coercivity of the '1st-input-2nd-output' operator $G_{21}$. We have conjectured that a so-called normal input realization of the lifted counterpart of $\Sigma\left(S^{\mathcal{V}}(\cdot), B_{1}, C_{1}\right)$ always generates a coercive $G_{21}$ operator. Such a realization exists for infinite-dimensional systems with the impulse response in $\ell_{1}\left(\mathbb{N}, \mathbb{R}^{p \times m}\right)$, for arbitrary finite $p$ and $m$; but it is not obvious under which assumptions it exists for infinite-dimensional discrete-time systems with arbitrary infinite-dimensional input and output spaces. For such systems, Ober \& Montgomery-Smith succeeded in proving only the existence of parabalanced realizations (see [26] for details). The development of such a realization theory for the general case of infinite-dimensional discrete-time systems with infinitedimensional input and output spaces would give-besides the insight in this part of realization theory-an adequate framework to formulate, in terms of initial data, sufficient conditions for the existence of a digital controller solving the $\mathrm{H}^{\infty}$ control problem.

\section{Acknowledgements}

The authors are extremely indebted to Prof. Ruth F. Curtain for the helpful suggestions and comments that led to a major improvement of the quality of this paper, and especially for drawing our attention on the way the digital results on $\mathcal{W}$ can appropriately be extended to $\mathcal{V}$.

\section{REFERENCES}

1. Aubin, J. P., 1979. Applied functional analysis. Wiley, New York.

2. BARB, F. D., 1994. Digital control of distributed parameter systems; a state-space approach. PhD thesis, Delft University of Technology.

3. Barb, F. D., IONescu, V., \& DE Koning, W. L., 1994. A Popov-theory-based approach to digital $\mathrm{H}^{x}$ control with measurement feedback for Pritchard-Salamon systems. IMA Journal of Mathematical Control \& Information 11, 277-309. 
4. Barb, F. D., \& De Koning, W. L., 1993. Digital control of Pritchard-Salamon systems. Report No. 33 (1993) of the faculty of Technical Mathematics and Informatics, Delft University of Technology. Submitted to International Journal of System Science.

5. Barb, F. D., DE Koning. W. L., \& Weiss, M., 1993. The digital linear quadratic control problem for Pritchard-Salamon systems. Proceedings of the 2nd European Control Conference ECC'93, Groningen, The Netherlands. Vol. 3, pp. 1371-76.

6. BARB, F. D., \& WEISs. M., 1993. Model reduction techniques for sampled-data systems. Numerical Algorithms 4, 47-64.

7. Bamieh, B. A., \& Pearson JR., J. B., 1992. A general framework for linear periodic systems with application to $\mathrm{H}^{x}$ sampled-data control. IEEE Transactions on Automatic Control 37, 418-35.

8. Bamieh, B., Pearson, J. B., Francis, B. A., \& Tannenbaum, A., 1991. A lifting technique for linear periodic systems with applications to sampled-data control. Systems \& Control Letters 17, 79-88.

9. BASAR, T., \& BERNhARD, P., 1991. $H^{x}$ optimal control and related minimax design problems: a dynamic game approach. Birkhäuser, Basel-Boston.

10. Bittanti, S., Laub, A. J., \& Willems, J. C., Eds., 1991. The Riccati equation. Springer.

11. Chen, T., \& Francis, B. A.. 1991. $\mathrm{H}^{2}$-optimal sampled-data control. IEEE Transactions on Automatic Control 36, 387-97.

12. Curtain, R., 1992. A synthesis of time-domain and frequency-domain methods for the control of infinite-dimensional systems: a system theoretic approach. Frontiers in Applied Mathematics (H. T. Banks, Ed.). Pp. 171-224.

13. Curtain, R., 1985. Sufficient conditions for infinite-rank Hankel operators to be nuclear. IM A Journal of Mathematical Control \& Information 2, 171-82.

14. Curtain, R., Logemann, H., Townley, S., \& Zwart, H., 1992. Well-posedness, stabilizability and admissibility for Pritchard-Salamon systems. Report No. 260-1 of the Institute of Dynamical Systems, University of Bremen. To appear in Journal of Mathematical Systems, Estimation and Control.

15. Delfour, M. $\dot{C}$., 1986. The linear quadratic control problem with delays in state and control variables: a state space approach. SIAM Journal on Control and Optimization 24, $835-83$.

16. Doyle, J. C., Glover, K., Francis, B. A., \& Khargonekar, P., 1989. State-space solutions to standard $\mathrm{H}^{2}$ and $\mathrm{H}^{\infty}$ control problems. IEEE Transactions on Automatic Control 34, 831-47.

17. Hager, W. W., \& Horowtrz, L. L., 1976. Convergence and stability properties of the discrete Riccati operator equation and the associated optimal control and filtering problems. SIAM Journal of Control \& Optimization 14, 295-312.

18. Halanay, A., \& Ionescu, V., 1993. Generalized discrete-time Popov-Yakubovitch theory. Systems \& Control Letters 20, 1-6.

19. Halanay, A., \& lonescu, V., 1994. Time-varying discrete linear systems, vol. OT.68 (I. Gohberg, Ed.). Birkhäuser.

20. IGLESIAS, P., 1991. State-space approach to discrete-time $\mathrm{H}^{x}$ control. International Journal of Control 54, 1031-73.

21. Ionescu, V., \& Weiss, M., 1993. Continuous and discrete-time Riccati theory: A Popov function approach. Linear Algebra and its Applications 193. Pp. 173-209.

22. Ionescu, V., \& Weiss, M., 1993. Two-Riccati formulae for discrete-time $\mathbf{H}^{x}$ control problem. International Journal of Control 57, 141-95.

23. Kailath, T., Vieira, A., \& MORF, M., 1978. Inverses of Toeplitz operators, innovations, and orthogonal polynomials. SIAM Review 20, 106-19.

24. Kalman, R. E., 1963. Lyapunov function for the problem of Lurie in automatic control. Proceedings in Mathematics of the Academy Sciences of the USA 49, 201-5.

25. LeE, K. Y., Chow, S. N., \& BarR, R. O., 1972. On the control of discrete-time distributed parameter systems. SIAM Journal of Control and Optimization 10, 361-76.

26. Öer, R., \& Montgomery-Sмiтh, S., 1990. Bilinear transformation of infinite-dimensional 
state-space systems and balanced realizations of nonrational transfer functions. SIAM Journal on Control and Optimization 28, 438-65.

27. Popov, V. M., 1973. Hyperstability of control systems. Editura Academiei Române, Bucharest; Springer Verlag, Berlin.

28. Pritchard, A., \& Salamon, D., 1987. The linear quadratic control problem for infinitedimensional systems with unbounded input and output operators. SIAM Journal of Control and Optimization 25, 121-44.

29. Rosen, I. G., \& WANG, C., 1992. On the continuous dependence with respect to sampling of the linear quadratic regulator problem for distributed parameter systems. SIAM Journal on Control and Optimization 30, 942-74.

30. SalAMON, D., 1987. Infinite dimensional linear systems with unbounded control and observation: a functional analytic approach. Transactions of the American Mathematical Society 300, 383-431.

31. Salamon, D., 1989. Realization theory in Hilbert space. Mathematical Systems Theory 21, 383-431.

32. StOORvogel, A., 1992. The $H^{\infty}$ control problem: a state-space approach. Prentice Hall.

33. TADMOR, G., 1992. $\mathrm{H}^{\infty}$ optimal sampled-data control in continuous time systems. International Journal of Control 56, 99-141.

34. VAN KEULEN, B., 1993. $H^{\infty}$-control for infinite-dimensional systems: a state-space approach. Prentice Hall.

35. WeIss, G., 1989. The represenatation of regular linear systems on Hilbert spaces. International series of Numerical Mathematics 91. Pp. 401-16.

36. WEIss, G., 1995. Regular linear systems with feedback. To appear in Mathematics of Control, Signals and Systems.

37. WeIss, M., 1994. Riccati equation on Hilbert space. A Popov function approach. Ph.D. thesis, University of Groningen, The Netherlands.

38. YaKUBOVITCH, V. A., 1962. Solutions of some matrix inequalities occurring in the theory of automatic control. Doklady Akademii Nauk SSSR 143(6).

39. Yамамото, Y., 1990. New approach to sampled-data control systems. A function space method. Proceedings of the 29th IEEE Conference on Decision and Control, Honolulu, Hawai, 1990. Pp. 1882-7. 